\title{
LIMIT THEOREMS FOR MEASURE-VALUED PROCESSES OF THE LEVEL-EXCEEDANCE TYPE
}

\author{
ANDRIY YURACHKIVSKY ${ }^{1}$
}

\begin{abstract}
Let, for each $t \in T, \psi(t, \cdot)$ be a random measure on the Borel $\sigma$-algebra in $\mathbb{R}^{d}$ such that $\mathrm{E} \psi\left(t, \mathbb{R}^{d}\right)^{k}<\infty$ for all $k$ and let $\widehat{\psi}(t, \cdot)$ be its characteristic function. We call the function $\widetilde{\psi}^{l}\left(t_{1}, \ldots, t_{l} ; z_{1}, \ldots, z_{l}\right)=\mathrm{E} \prod_{j=1}^{l} \widehat{\psi}\left(t_{j}, z_{j}\right)$ of arguments $l \in \mathbb{N}, t_{1}, t_{2} \ldots \in T, z_{1}, z_{2} \ldots \in \mathbb{R}^{d}$ the covaristic of the measure-valued random function (MVRF) $\psi(\cdot, \cdot)$. A general limit theorem for MVRF's in terms of covaristics is proved and applied to functions of the kind $\psi_{n}(t, B)=\mu\left\{x: \xi_{n}(t, x) \in B\right\}$, where $\mu$ is a nonrandom finite measure and, for each $n, \xi_{n}$ is a time-dependent random field.
\end{abstract}

Mathematics Subject Classification. 60G57,60F17.

Received March 18, 2009. Revised November 17, 2009.

\section{INTRODUCTION}

Let $(\Omega, \mathcal{F}, \mathrm{P})$ be a probability space, $(X, \mathcal{X})$ and $(S, \mathcal{S})$ be measurable spaces. An $\mathcal{X} \otimes \mathcal{F} \mid \mathcal{S}$-measurable function $\xi: X \times \Omega \rightarrow S$ will be called an $S$-valued random relief on $X$. The argument $\omega \in \Omega$ of this and other functions will be usually omitted. If a measure $\mu$ on $\mathcal{X}$ is given, then a random relief $\xi$ induces the random measure

$$
\mu^{\xi}(B) \stackrel{\text { def }}{=} \mu\{x: \xi(x) \in B\}
$$

on $\mathcal{S}$. In case $S=\mathbb{R}$ it is called the level-exceedance measure of the random field (in our terminology - relief) $\xi$. In case $S$ is arbitrary we will say that $\mu^{\xi}$ is a random measure of the level-exceedance type.

A random relief depending additionally on a parameter (which may and will perform as a variable) will be called varying. Such a relief induces, together with $\mu$, a measure-valued random function (MVRF).

The goals of the article are to provide a tool for proving functional limit theorems for MVRF's (this is accomplished in Sect. 2) and to apply it to measure-valued processes of the level-exceedance type arising in some model related to stochastic geometry (Sect. 3). Proofs of the theorems of Section 3 are placed to Section 4. Section 1 contains some general theorems about random measures and measure-valued functions.

The theory of random measures and measure-valued processes is well developed $[1-3,8,16]$. But the existing methods of this theory do not provide sufficiently general functional limit theorems for the processes we are going to consider. Our approach is based on the use of the Fourier-Stieltjes transform of moment measure (more precisely, some generalization of the latter). In these terms, we shall prove two functional analogues of Lévy's continuity theorem (see Thms. 2.3 and 2.4 below) and demonstrate their application.

\footnotetext{
Keywords and phrases. Measure-valued process, covaristic, convergence, relative compactness

1 Taras Shevchenko National University, vul. Volodymyrska 64, Kyiv, 01601, Ukraine. yap@univ.kiev.ua
} 


\section{UNIVERSAL CONSTRUCTIONS OF MEASURE-VALUED RANDOM FUNCTIONS}

In what follows, $\mathcal{B}(X)$ signifies the $\sigma$-algebra of Borel sets in a topological space $X ; \mathcal{B}$ means $\mathcal{B}(\mathbb{R})$ (so that $\left.\mathcal{B}\left(\mathbb{R}^{d}\right)=\mathcal{B}^{\otimes d}\right), \mathcal{B}_{+}$stands for $\mathcal{B}\left(\mathbb{R}_{+}\right) ; \mathrm{C}_{\mathrm{b}}(X)$ is the set of all bounded continuous functions on $X$.

If $(S, \mathcal{S})$ is a measurable space, then $\mathbb{M}_{+}(S), \mathbb{M}(S)$ and $\mathbb{P}(S)$ signify the collections of: all (finite) measures on $\mathcal{S}$, all signed measures on $\mathcal{S}$ and all probability measures on $\mathcal{S}$, respectively. The $\sigma$-algebra in $\mathbb{M}_{+}(S)$ generated by the sets

$$
H_{B}^{A}=\left\{m \in \mathbb{M}_{+}(S): m(A) \in B\right\}, A \in \mathcal{S}, B \in \mathcal{B}_{+},
$$

will be denoted $\mathfrak{M}(\mathcal{S})$. In case $S$ is a topological space we equip $\mathbb{M}(S)$ with the topology generated by the sets $\left\{q \in \mathbb{M}(S): \int f \mathrm{~d} q \in U\right\}$, where $f$ and $U$ range over $\mathrm{C}_{\mathrm{b}}(S)$ and the collection of open sets in $\mathbb{R}$, respectively. In functional analysis, this topology is called $* w e a k$; in probability theory, its restriction to $\mathbb{M}_{+}(S)$ is called, somewhat loosely, the weak topology. The latter unlike the former is, under rather general assumptions, metrisable ([17], Proposition 1.3.11; this result is attributed in [17] to Prokhorov, but what he did prove in [11] is metrisability of weak convergence). We call a topological space $S$ concordant if $\mathfrak{M}(\mathcal{S})=\mathcal{B}\left(\mathbb{M}_{+}(S)\right)$, where $\mathcal{S}$ is the $\sigma$-algebra of Baire sets in $S$.

The following statement asserts that a random measure of the level-exceedance type is an $\mathbb{M}_{+}$-valued random element.

Lemma 1.1. Let $Y$ be a concordant topological space, $(\Omega, \mathcal{F})$ be a measurable space and $v$ be a function on $\Omega \times \mathcal{B}(Y)$ such that for any $\omega \in \Omega \quad v(\omega, \cdot) \in \mathbb{M}_{+}(Y)$ and for any $A \in \mathcal{B}(Y) \quad v(\cdot, A)$ is $\mathcal{F} \mid \mathcal{B}$-measurable. Then $\{\omega: v(\omega, \cdot) \in D\} \in \mathcal{F}$ for all $D \in \mathcal{B}\left(\mathbb{M}_{+}(Y)\right)$.

Proof. By assumption this is true for all $D=H_{B}^{A}(A \in \mathcal{B}(Y), B \in \mathcal{B})$ and therefore, by the definition of $\mathfrak{M}(Y)$, for all its elements.

Remark 1.1. The class of concordant topological spaces is vast. Theorem A.2.6 in [2] asserts that it contains all locally compact Polish spaces, in particular $\mathbb{R}^{d}$. One can prove that it contains even all metric spaces. We shall not use this fact.

Henceforth $\mathcal{X}$ and $\mathcal{Y}$ stand for $\mathcal{B}(X)$ and $\mathcal{B}(Y)$, respectively. We remind that a measure is called diffuse if it equals zero at each singleton.

Theorem 1.1. Let $Y$ be a concordant separable complete metric space, $(\Omega, \mathcal{F})$ be a measurable space and $\Psi$ be an $\mathcal{F} \mid \mathfrak{M}(\mathcal{Y})$-measurable map from $\Omega$ to $\mathbb{P}(Y)$. Then for any separable complete metric space $X$ and any diffuse probability measure $\mu$ on $\mathcal{X}$ there exists an $\mathcal{X} \otimes \mathcal{F} \mid \mathcal{Y}$-measurable map $\xi: X \times \Omega \rightarrow Y$ such that $\Psi=\mu^{\xi}$.

We prove first a particular case of the theorem, formulating it in the autonomous notation.

Lemma 1.2. Let $\nu$ be a probability measure on the Borel $\sigma$-algebra of a separable complete metric space $Z$. Then for any separable complete metric space $X$ and any diffuse probability measure $\mu$ on $\mathcal{X}$ there exists a Borel function $f: X \rightarrow Z$ such that $\nu=\mu \circ f^{-1}$.

Proof. By Lemma 4.1.1 in [4] there exists a Borel function $g: X \rightarrow[0,1]$ such that $g\left(x_{1}\right) \neq g\left(x_{2}\right)$ as $x_{1} \neq x_{2}$. Denote $m=\mu \circ g^{-1}, F(u)=m([0, u])(=0$ as $u<0), \widetilde{F}(v)=\sup \{u \in[0,1]: F(u) \leq v\}$. For any $u \in \mathbb{R}$, $\mu\left(g^{-1}\{u\}\right)=0$, because, by the choice of $g$, the set $g^{-1}\{u\}$ contains either one element or none and the measure $\mu$ is, by assumption, diffuse. So $F$ is continuous and therefore $F(\widetilde{F}(v))=v$. But $F(\widetilde{F}(v))=m\left(F^{-1}[0, v]\right)$. Consequently, $m \circ F^{-1}=\lambda$, where $\lambda$ is the Lebesgue measure on $\mathcal{B}[0,1]$. Juxtaposing this with the definition of $m$, we see that

$$
\mu \circ(F \circ g)^{-1}=\lambda \text {. }
$$

Skorokhod's principle of a common probability space [13,14] asserts, in particular, existence of a Borel function $h:[0,1] \rightarrow Z$ such that $\nu=\lambda \circ h^{-1}$. Comparing this with (1.1), we see that $\nu=\mu \circ(h \circ F \circ g)^{-1}$. 
Proof of Theorem 1.1. For $X=[0,1]$ and $\mu=\lambda$ this is Theorem 1.2.1 in [15]. Consequently, there exists a $\mathcal{B}[0,1] \otimes \mathcal{F} \mid \mathcal{Y}$-measurable map $\zeta:[0,1] \times \Omega \rightarrow Y$ such that for any $B \in \mathcal{Y}$

$$
\psi(B)=\lambda\{z: \zeta(z) \in B\} .
$$

Evidently, for any $B \subset Y$ and $q \in[0,1]^{X}$

$$
q^{-1}\{z: \zeta(z) \in B\}=\{x: \zeta(q(x)) \in B\} .
$$

Lemma 1.2 asserts existence of a Borel function $f: X \rightarrow[0,1]$ such that $\mu \circ f^{-1}=\lambda$. So, putting in (1.3) $q=f$, we get for any $B \in \mathcal{Y}$

$$
\lambda\{z: \zeta(z) \in B\}=\mu\{x: \zeta(f(x)) \in B\} .
$$

It remains, in view of $(1.2)$, to set $\xi=\zeta \circ f$.

Wishing to discern a measure valued-function and its value on a set, we use throughout the notation $\Psi(t)=$ $\psi(t, \cdot), \Upsilon(t)=v(t, \cdot)$ which will not be explained further.

Corollary 1.1. Let $Y$ be a concordant separable complete metric space and $\Psi$ be a $\mathbb{P}(Y)$-valued random function on some set $T$ given on some probability space. Then for any separable complete metric space $X$ and any diffuse probability measure $\mu$ on $\mathcal{X}$ there exists a $Y$-valued varying random relief $\xi$ on $X$ given on the same probability space and such that for all $t \in T$ and $B \in \mathcal{Y}$

$$
\psi(t, B)=\mu\{x: \xi(t, x) \in B\}
$$

Let $(S, \mathcal{S})$ be an arbitrary measurable space and $\Psi$ be an $\mathbb{M}_{+}(S)$-valued random function on some infinite set $T$. We denote

$$
\bar{\psi}^{l}\left(t_{1}, \ldots, t_{l} ; A_{1}, \ldots, A_{l}\right)=\mathrm{E} \prod_{j=1}^{l} \psi\left(t_{j}, A_{j}\right)
$$

and call the $\mathbb{R}_{+} \cup\{\infty\}$-valued function $\bar{\psi}$ of variables $l \in \mathbb{N}, t_{1}, t_{2} \ldots \in T\left(t_{i} \neq t_{j}\right.$ as $\left.i \neq j\right), A_{1}, A_{2} \ldots \in \mathcal{S}$ the multiplex of $\Psi$. The likewise defined and denoted function on $\mathbb{N} \times T^{\mathbb{N}} \times \mathcal{S}^{\mathbb{N}}$ (the equality $t_{i}=t_{j}$ is allowed and $T$ may be finite) will be called the extended multiplex of $\Psi$. The multiplex (in both variants) can be identified with the sequence $\left(\bar{\psi}^{l}, l \in \mathbb{N}\right)$ whose $l$ th member is a function on $\mathbf{T} \times \mathcal{S}^{l}, \mathbf{T} \subset T^{l}$. The short notation of this sequence will be $\bar{\psi}$.

Theorem 1.2. Let a separable complete metric space $Y$, an infinite set $T$ and, for each $l \in \mathbb{N}$, an $\mathbb{R}$-valued function $\Lambda^{l}$ of variables $t_{1} \in T, t_{2} \in T \backslash\left\{t_{1}\right\}, \ldots, t_{l} \in T \backslash\left\{t_{1}, \ldots, t_{l-1}\right\}, A_{1} \in \mathcal{Y}, \ldots, A_{l} \in \mathcal{Y}$ be given. Suppose that these functions have the properties: (a) $\Lambda^{l}\left(t_{1}, \ldots, t_{l} ; A_{1}, \ldots, A_{l-1}, \cdot\right) \in \mathbb{M}_{+}(Y)$;

(b) for any permutation $\left(\begin{array}{ccc}1 & \ldots & l \\ i_{1} & \ldots & i_{l}\end{array}\right)$

$$
\Lambda^{l}\left(t_{i_{1}}, \ldots, t_{i_{l}} ; A_{i_{1}}, \ldots, A_{i_{l}}\right)=\Lambda^{l}\left(t_{1}, \ldots, t_{l} ; A_{1}, \ldots, A_{l}\right) ;
$$

(c) $\Lambda^{l}\left(t_{1}, \ldots, t_{l} ; A_{1}, \ldots, A_{l-1}, Y\right)=\Lambda^{l-1}\left(t_{1}, \ldots, t_{l-1} ; A_{1}, \ldots, A_{l-1}\right)(l>1) ;(d) \Lambda^{1}(t ; Y)=1$.

Then there exists a $\mathbb{P}(Y)$-valued random function $\Upsilon$ on $T$ such that $\Lambda$ is its multiplex.

Proof. Properties (a)-(d) together with separability and completeness of $Y$ imply, by Kolmogorov's theorem, existence of a $Y$-valued random function $\zeta$ on $T$ such that for any $l \in \mathbb{N}, t_{1} \in T, t_{2} \in T \backslash\left\{t_{1}\right\}, \ldots, t_{l} \in$ $T \backslash\left\{t_{1}, \ldots, t_{l-1}\right\} \quad A_{1} \in \mathcal{Y}, \ldots, A_{l} \in \mathcal{Y}$

$$
\mathrm{P}\left\{\zeta\left(t_{1}\right) \in A_{1}, \ldots, \zeta\left(t_{l}\right) \in A_{l}\right\}=\Lambda^{l}\left(t_{1}, \ldots, t_{l} ; A_{1}, \ldots, A_{l}\right) .
$$


Put

$$
v(t, A)= \begin{cases}1 & \text { if } \zeta(t) \in A \\ 0 & \text { otherwise }\end{cases}
$$

Then $v(t, \cdot)$ is a random probability measure on $\mathcal{Y}$ and

$$
\bar{v}^{l}\left(t_{1}, \ldots, t_{l} ; A_{1}, \ldots, A_{l}\right)=\mathrm{P}\left\{\zeta\left(t_{1}\right) \in A_{1}, \ldots, \zeta\left(t_{l}\right) \in A_{l}\right\} .
$$

Theorem 1.3. Let $Y$ be a separable complete metric space, $T$ be an infinite set and, for each $l \in \mathbb{N}, \Lambda^{l}$ be an $\mathbb{R}$-valued function on $T^{l} \times \mathcal{Y}^{l}$. Suppose that the functions $\Lambda^{l}$ have properties (a)-(d) and one more property

$$
\Lambda^{l}\left(t_{1}, \ldots, t_{l-1}, t_{l-1} ; A_{1}, \ldots, A_{l}\right)=\Lambda^{l-1}\left(t_{1}, \ldots, t_{l-1} ; A_{1}, \ldots, A_{l-2}, A_{l-1} \cap A_{l}\right) .
$$

Then there exists a $\mathbb{P}(Y)$-valued random function $\Upsilon$ on $T$ such that $\Lambda$ is its extended multiplex.

Proof. Let us construct $\zeta$ and $\Upsilon$ as in the proof of Theorem 1.2. Then equalities (1.4) and (1.6) hold for all $A_{1}, \ldots, A_{l} \in \mathcal{Y}$ and pairwise different $t_{1}, \ldots, t_{l} \in T$. Besides that, by the construction of $v$

$$
\bar{v}^{l}\left(t_{1}, \ldots, t_{l-1}, t_{l-1} ; A_{1}, \ldots, A_{l}\right)=\bar{v}^{l-1}\left(t_{1}, \ldots, t_{l-1} ; A_{1}, \ldots, A_{l-2}, A_{l-1} \cap A_{l}\right) .
$$

This together with condition (1.7) shows that equalities (1.4) and (1.6) remain valid in case some of the points $t_{1}, \ldots, t_{l}$ coincide.

Remark 1.2. The analogue of Theorems 1.2 and 1.3 for the case when $T$ is a singleton (so that MVRF becomes simply random measure and multiplex becomes moment function [2,8,16,26]) was proved in [22]. It contains one more condition and its proof based on convex analysis is by far more difficult.

Let $(S, \mathcal{S})$ be an arbitrary measurable space. We call an $\mathbb{M}_{+}(S)$-valued random function $\Psi$ on $T$ momentable if its extended multiplex assumes only finite values and determines the finite-dimensional distributions of $\Psi$. Recalling the definition of the $\sigma$-algebra $\mathfrak{M}(\mathcal{S})$, we see that the second demand is tantamount to the property that the extended multiplex determines the probabilities $\mathrm{P}\left\{\Psi\left(t_{1}\right) \in H_{B_{1}}^{A_{1}}, \ldots, \Psi\left(t_{l}\right) \in H_{B_{l}}^{A_{l}}\right\}$ for all possible $l \in \mathbb{N}, A_{1}, \ldots, A_{l} \in \mathcal{S}$ and pairwise different $t_{1}, \ldots, t_{l} \in T$. But the relation $\Psi(t) \in H_{B}^{A}$ means that $\psi(t, A) \in B$. So $\Psi$ is momentable if and only if its extended multiplex is finite and determines the distributions of all the vectors $\left(\psi\left(t_{1}, A_{1}\right), \ldots, \psi\left(t_{l}, A_{l}\right)\right), l \in \mathbb{N}, t_{j} \in T, A_{j} \in \mathcal{S}$ (it may seem that we must add " $t_{i} \neq t_{j}$ as $i \neq j$ ", but, obviously, this restriction in this context is redundant).

Denote $\Xi(t)=\psi(t, S)$.

Lemma 1.3. Let $\Psi$ be an $\mathbb{M}_{+}(S)$-valued random function on $T$ such that for all $k \in \mathbb{N}$ and $t_{1}, \ldots, t_{k} \in T$

$$
\sum_{l=1}^{\infty}\left(\sum_{i=1}^{k} \mathrm{E} \Xi\left(t_{i}\right)^{l}\right)^{-\frac{1}{2 l}}=\infty
$$

Then $\Psi$ is momentable.

Proof. Let us take arbitrary positive numbers $a_{1}, \ldots, a_{k}$ and denote $\beta_{i}=\psi\left(t_{i}, A_{i}\right), \alpha=\sum_{i=1}^{k} a_{i} \beta_{i}$. By Hölder's inequality

whence

$$
\alpha^{l} \leq\left(\sum_{i=1}^{k} a_{i}^{l /(l-1)}\right)^{l-1} \sum_{i=1}^{k} \beta_{i}^{l}
$$

$$
\mathrm{E} \alpha^{l} \leq k^{l-1} \max _{i} a_{i}{ }^{l} \sum_{i=1}^{k} \mathrm{E} \Xi\left(t_{i}\right)^{l} .
$$


Then (1.8) yields $\sum\left(\mathrm{E} \alpha^{l}\right)^{-1 / 2 l}=\infty$. This means, by Carleman's theorem ([12], Sect. II.12) that the distribution of the random variable $\alpha$ (and, consequently, the value of $\mathrm{Ee}^{-\alpha}$ ) is determined by its moments. And they, in turn, are determined by the extended multiplex of $\Psi$.

Corollary 1.2. Any probability-valued random function is momentable.

Corollary 1.3. Let $Y$ be a separable complete metric space and $\Psi$ be a $\mathbb{P}(Y)$-valued random function on some infinite set $T$. Then there exist a Markov kernel $P=P(y, A)(y \in Y, A \in \mathcal{Y})$ and a $Y$-valued random function $\zeta$ on $T$ such that $\Psi$ has the same finite-dimensional distributions as $\Upsilon$, where

$$
v(t, A)=P(\zeta(t), A)
$$

Proof. Setting in Theorem $1.3 \Lambda=\bar{\psi}$, where $\bar{\psi}$ is the extended multiplex of $\Psi$, we conclude from its proof that $\bar{\psi}=\bar{v}$, where $v(t, A)$ is defined by equality (1.5). And the last is a particular case $\left(P(y, A)=I_{A}(y)\right)$ of $(1.9)$. It remains to refer to Corollary 1.2.

Remark 1.3. Corollary 1.3 sustains if the set $T$ is finite. For the case when $T$ is a singleton it was proved in [22]. In this case (as well as for any finite $T$ ) the representation (1.9) need not perform in its simplest form $(1.5)$.

\section{THE TOOL}

We shall denote the characteristic function of a measure on $\mathcal{B}^{\otimes d}$ in the following manner:

$$
\widehat{m}(z)=\int \mathrm{e}^{\mathrm{i} z y} m(\mathrm{~d} y) .
$$

Here $\int=\int_{\mathbb{R}^{d}}, z \in \mathbb{R}^{d}$ is a row vector (and other vectors are meant as columns). Henceforth "measure-valued" means "M $\mathbb{M}_{+}\left(\mathbb{R}^{d}\right)$-valued" and "probability-valued" means " $\mathbb{P}\left(\mathbb{R}^{d}\right)$-valued".

We call the function

$$
\widetilde{v}^{l}\left(t_{1}, \ldots, t_{l} ; z_{1}, \ldots, z_{l}\right)=\mathrm{E} \prod_{j=1}^{l} \widehat{v}\left(t_{j}, z_{j}\right)
$$

of variables $l \in \mathbb{N}, t_{1}, t_{2} \ldots \in T, z_{1}, z_{2} \ldots \in \mathbb{R}^{d}$ the covaristic (the short term for covariance-characteristic function) of a momentable MVRF $\Upsilon$ on $T$. This notion was introduced first for independent of $t$ random probability measures in $[18,20]$, then for probability-valued random functions in [19] and at last, in the present form, in [23] where the following statement was proved.

Theorem 2.1. The covaristic of a momentable MVRF determines its finite-dimensional distributions.

Let $\Upsilon$ be a momentable MVRF. Then, for each $l, t_{1}, \ldots, t_{l}$, the function $\bar{v}^{l}\left(t_{1}, \ldots, t_{l} ; \cdot, \ldots, \cdot\right)$ induces a measure on $\mathcal{B}^{\otimes d l}$. This together with the definition of multiplex and Fubini's theorem yields an equivalent form of equality (2.1):

$$
\widetilde{v}^{l}\left(t_{1}, \ldots, t_{l} ; z_{1}, \ldots, z_{l}\right)=\widehat{\bar{v}}^{l}\left(t_{1}, \ldots, t_{l} ; z_{1}, \ldots, z_{l}\right) \equiv \int \ldots \int \exp \left(i \sum_{j=1}^{l} z_{j} y_{j}\right) \bar{v}^{l}\left(t_{1}, \ldots, t_{l} ; \mathrm{d} y_{1}, \ldots, \mathrm{d} y_{l}\right)
$$

We shall identify the covaristic of a momentable MVRF $\Upsilon$ with the sequence $\left(\widetilde{v}^{l}\right)$ whose $l$ th member is defined by $(2.1)$. 
Theorem 2.2. Let $T$ be an infinite set and, for $l=1,2, \ldots, \Phi^{l}$ be $a \mathbb{C}$-valued function on $T^{l} \times \mathbb{R}^{d l}$ such that: $\Phi^{l}\left(t_{1}, \ldots, t_{l} ; \cdot, \ldots, \cdot\right)$ is positive definite,

$$
\lim _{z_{1} \rightarrow 0, \ldots, z_{l} \rightarrow 0} \Phi^{l}\left(t_{1}, \ldots, t_{l} ; z_{1}, \ldots, z_{l}\right)=1
$$

and for any permutation $\left(\begin{array}{ccc}1 & \ldots & l \\ i_{1} & \ldots & i_{l}\end{array}\right)$

$$
\Phi^{l}\left(t_{i_{1}}, \ldots, t_{i_{l}} ; z_{i_{1}}, \ldots, z_{i_{l}}\right)=\Phi^{l}\left(t_{1}, \ldots, t_{l} ; z_{1}, \ldots, z_{l}\right) .
$$

Suppose also that

$$
\Phi^{1}(t ; 0)=1
$$

and for any $l \geq 2, t_{1}, \ldots, t_{l}, z_{1}, \ldots, z_{l}$

$$
\Phi^{l}\left(t_{1}, \ldots, t_{l} ; z_{1}, \ldots, z_{l-1}, 0\right)=\Phi^{l-1}\left(t_{1}, \ldots, t_{l-1} ; z_{1}, \ldots, z_{l-1}\right) .
$$

Then there exists a probability-valued random function $\Upsilon$ on $T$ such that

$$
\widetilde{v}^{l}\left(t_{1}, \ldots, t_{l} ; z_{1}, \ldots, z_{l}\right)=\Phi^{l}\left(t_{1}, \ldots, t_{l} ; z_{1}, \ldots, z_{l}\right)
$$

for all $l \in \mathbb{N}, t_{1} \in T, t_{2} \in T \backslash\left\{t_{1}\right\}, \ldots, t_{l} \in T \backslash\left\{t_{1}, \ldots, t_{l-1}\right\}, z_{1}, \ldots, z_{l} \in \mathbb{R}^{d}$.

Proof. Denote $\mathbf{t}=\left(t_{1}, \ldots, t_{l}\right), \mathbf{z}=\left(z_{1}, \ldots, z_{l}\right)$. The first two assumptions imply, by the Bochner-Khinchin theorem, existence of a probability measure $P^{l}(\mathbf{t} ; \cdot)$ on $\mathcal{B}^{\otimes d l}$ such that

$$
\Phi^{l}(\mathbf{t} ; \mathbf{z})=\int_{\mathbb{R}^{d l}} \mathrm{e}^{\mathrm{i} \mathbf{z y}} P^{l}(\mathbf{t} ; \mathrm{d} \mathbf{y}) .
$$

Denote

$$
\Lambda^{l}\left(t_{1}, \ldots, t_{l} ; A_{1}, \ldots, A_{l}\right)=P^{l}\left(\mathbf{t} ; A_{1} \times \ldots \times A_{l}\right) .
$$

Obviously, this function has property (a) from Theorem 1.2. Equality (2.3) shows that the sequence $\left(\Lambda^{l}\right)$ has property (b). Equalities (2.5), (2.7) and (2.8) yield property (c). Finally, (d) is immediate from (2.4), (2.7) and (2.8). Then Theorem 1.2 asserts existence of a probability-valued random function $\Upsilon$ on $T$ such that

$$
P^{l}\left(\mathbf{t} ; A_{1} \times \ldots \times A_{l}\right)=\bar{v}^{l}\left(\mathbf{t} ; A_{1}, \ldots, A_{l}\right) .
$$

Substituting this to (2.7) and taking to account (2.2), we arrive at (2.6).

Remark 2.1. The analogue of Theorem 2.2 for the case when $T$ is a singleton (so that MVRF becomes simply random measure) was proved in [22]. It contains an extra condition, and its proof is more complicated.

Corollary 2.1. Let the conditions of Theorem 2.2 be fulfilled. Then for any separable complete metric space $X$ and any diffuse probability measure $\mu$ on $\mathcal{X}$ there exists an $\mathbb{R}^{d}$-valued varying random relief $Q$ on $X$ such that

$$
\Phi^{l}\left(t_{1}, \ldots, t_{l} ; z_{1}, \ldots, z_{l}\right)=\mathrm{E} \prod_{j=1}^{l} \int \mathrm{e}^{\mathrm{i} z_{j} Q\left(t_{j}, x\right)} \mu(\mathrm{d} x) .
$$

Proof. Corollary 1.1 asserts existence of a varying random relief $Q$ such that $v(t, B)=\mu\{x: Q(t, x) \in B\}$. Hence $\widehat{v}(t, z)=\int \mathrm{e}^{\mathrm{i} z Q(t, x)} \mu(\mathrm{d} x)$, which together with (2.1) and (2.6) yields (2.9). 
Let $Y$ be a metric space. We denote

$$
\mathfrak{F}=\left\{\varphi \in \mathbb{R}^{Y}: \forall y, y^{\prime} \in Y|\varphi(y)| \leq 1 \&\left|\varphi(y)-\varphi\left(y^{\prime}\right)\right| \leq \operatorname{dist}\left(y, y^{\prime}\right) \wedge 1\right\}
$$

and equip $\mathbb{M}(Y)$ with the bounded Lipschitz seminorm $\|q\|_{B L}=\sup _{\varphi \in \mathfrak{F}}\left|\int \varphi \mathrm{d} q\right|$. The respective pseudometric will be denoted $d_{B L}$.

Lemma 2.1. Let $Y$ be a separable complete metric space. Then: (1) $d_{B L}$ is a metric in $\mathbb{M}_{+}(Y)$; (2) the metric space $\left(\mathbb{M}_{+}(Y), d_{B L}\right)$ is complete; (3) the convergence in this metric space coincides with the weak one.

Proof. Since any finite measure is proportional to a probability measure, all the three statements are easy consequences of the well-known similar properties of $\mathbb{P}_{+}(Y)$ (see, e.g., Thms. 2.3.8 and 2.3.9, Lem. 2.4.1 and Cor. 2.4.3 in [6]).

Remark 2.2. The second and the third statement of Lemma 2.1 do not carry over to the space $\mathbb{M}(Y)$ !

We use the following notation of convergences: $\stackrel{\mathrm{d}}{\longrightarrow}$ means weak convergence of finite-dimensional distributions of $\mathbb{R}^{k}$-valued or $\mathbb{C}^{k}$-valued random functions (whose argument may take values in a finite set as well as in an infinite one); $\stackrel{d}{\longrightarrow}$ means the same for measure-valued functions. If $\alpha_{1}, \alpha_{2}, \ldots, \alpha$ are $\mathbb{R}^{k}$ - or $\mathbb{C}^{k}$-valued càdlàg random processes such that $\alpha$ is continuous and $\phi\left(\alpha_{n}\right) \stackrel{\mathrm{d}}{\longrightarrow} \phi(\alpha)$ for any continuous in the locally uniform topology functional $\phi$ on the Skorokhod space D, then we write $\alpha_{n} \stackrel{\mathrm{C}}{\longrightarrow} \alpha$. We say that a sequence $\left(\alpha_{n}\right)$ is relatively compact (r.c.) in $\mathrm{C}$ if each its subsequence contains, in turn, a subsequence convergent in the above sense. This terminology carries over to measure-valued processes (MVP) with $\mathcal{C}$ instead of $\mathrm{C}$ in notation.

We also denote $A_{L}=\left\{x \in \mathbb{R}^{d}:|x|>L\right\}$. The choice of norm in $\mathbb{R}^{d}$ is, as will be seen from the conditions where $A_{L}$ performs, inessential.

Theorem 2.3. Let $\left(\Psi_{n}\right)$ be a sequence of $M V P^{\prime}$ 's such that: for any $l \in \mathbb{N}, t_{1}, \ldots, t_{l} \in \mathbb{R}_{+}, z_{1}, \ldots, z_{l} \in \mathbb{R}^{d}$

$$
\widetilde{\psi}_{n}^{l}\left(t_{1}, \ldots, t_{l} ; z_{1}, \ldots, z_{l}\right) \rightarrow U^{l}\left(t_{1}, \ldots, t_{l} ; z_{1}, \ldots, z_{l}\right)
$$

for any $k \in \mathbb{N}, s_{1}, \ldots, s_{k}, t \in \mathbb{R}_{+}$

$$
\begin{gathered}
\sum_{l=1}^{\infty}\left(\sum_{j=1}^{k} U^{l}\left(s_{j}, \ldots, s_{j} ; 0, \ldots, 0\right)\right)^{-\frac{1}{2 l}}=\infty \\
\sup _{n} E \Xi_{n}(t)^{k}<\infty
\end{gathered}
$$

for any $t \in \mathbb{R}_{+}$

$$
\lim _{z \rightarrow 0} U^{1}(t ; z)=U^{1}(t ; 0)
$$

for any $t>0, \varepsilon>0$ and $z \in \mathbb{R}^{d}$

$$
\begin{gathered}
\lim _{L \rightarrow \infty} \varlimsup_{n \rightarrow \infty} \sup _{s \leq t} \mathrm{P}\left\{\Xi_{n}(s)>L\right\}=0, \\
\lim _{L \rightarrow \infty} \varlimsup_{n \rightarrow \infty} \sup _{s \leq t} \mathrm{P}\left\{\psi_{n}\left(s, A_{L}\right)>\varepsilon\right\}=0, \\
\lim _{c \rightarrow \infty} \varlimsup_{n \rightarrow \infty} \sup _{(v-c)_{+} \leq u<v \leq t} \mathrm{P}\left\{\left|\widehat{\psi}_{n}(u, z)-\widehat{\psi}_{n}(v, z)\right|>\varepsilon\right\}=0 .
\end{gathered}
$$

Then $U$ is the covaristic of some momentable MVP $\Psi$ and $\Psi_{n} \stackrel{d}{\longrightarrow} \Psi$. 
Proof. Let $T$ be an arbitrary countable dense set in $\mathbb{R}_{+}$. Theorem 2 [23] asserts that under conditions (2.10)(2.13) where $t_{1}, \ldots, t_{l}, s_{1}, \ldots, s_{k}$ and $t$ are taken from $T$ there exists an MVRF $\Psi$ on $T$ such that the conclusion of the theorem holds on $T$. Consequently, for any $k \in \mathbb{N}, t_{1}, \ldots, t_{k} \in T$ and bounded continuous functional $\phi$ on $\left(\mathbb{M}_{+}\left(\mathbb{R}^{d}\right)\right)^{k}$

$$
\mathrm{E} \phi\left(\Psi_{n}\left(t_{1}\right), \ldots, \Psi_{n}\left(t_{k}\right)\right) \rightarrow \mathrm{E} \phi\left(\Psi\left(t_{1}\right), \ldots, \Psi\left(t_{k}\right)\right) .
$$

To assert the same for arbitrary $t_{1}, \ldots, t_{k} \in \mathbb{R}_{+}$it suffices, in the light of Lemma 2.1, to show that for all $t>0, \varepsilon>0$

$$
\lim _{c \rightarrow \infty} \varlimsup_{n \rightarrow \infty} \sup _{(v-c)_{+} \leq u<v \leq t} \mathrm{P}\left\{\left\|\Psi_{n}(u)-\Psi_{n}(v)\right\|_{B L}>\varepsilon\right\}=0 .
$$

Indeed, relation (2.18) ensures uniform in any interval stochastic continuity of $\Psi$ and thus allows to uniquely extend $\Psi$ to a stochastically continuous MVP on $\mathbb{R}_{+}$, thereupon (2.17) carries over, again due to (2.18), from $T$ to $\mathbb{R}_{+}$.

Let us equip $\mathfrak{F}$ (defined for $Y=\mathbb{R}^{d}$ ) with the metric

$$
\delta(f, g)=\sum_{L=1}^{\infty} 2^{-L} \max _{|x| \leq L}|f(x)-g(x)| .
$$

Functions from $\mathfrak{F}$ being uniformly bounded and equicontinuous, each sequence of its members contains a subsequence converging uniformly on each compact set. So for any $a>0$ there exists a finite $a$-mesh in $\mathfrak{F}$.

Let us fix $f \in \mathfrak{F}, L \in \mathbb{N}$ and $a$-mesh $\left\{g_{1}, \ldots, g_{r}\right\}$. We choose $g \in\left\{g_{1}, \ldots, g_{r}\right\}$ such that $\delta(f, g)<a$ and, for each $j \in\{1, \ldots, r\}$, take a trigonometric polynomial $p_{j}$ such that $\sup _{|x| \leq L}\left|g_{j}(x)-p_{j}(x)\right|<a$ (the $p_{j}$ corresponding to $g$ will be written without subscript). Denote $C=\|g-p\|_{\infty}$. Then for any finite measure $m$

$$
\int|f-g| \mathrm{d} m \leq 2^{L} a m\left(\mathbb{R}^{d}\right)+2 m\left(A_{L}\right), \quad \int|g-p| \mathrm{d} m \leq a m\left(\mathbb{R}^{d}\right)+C m\left(A_{L}\right) .
$$

These inequalities together with the identity

$$
\int f \mathrm{~d} m_{1}-\int f \mathrm{~d} m_{2}=\int(f-g) \mathrm{d} m_{1}-\int(f-g) \mathrm{d} m_{2}+\int(g-p) \mathrm{d} m_{1}-\int(g-p) \mathrm{d} m_{2}+\int p \mathrm{~d} m_{1}-\int p \mathrm{~d} m_{2}
$$

imply that

$$
\left\|m_{1}-m_{2}\right\| \leq \sum_{i=1}^{2}\left(\left(2^{L}+1\right) a m_{i}\left(\mathbb{R}^{d}\right)+(C+2) m_{i}\left(A_{L}\right)\right)+\max _{1 \leq j \leq r}\left|\int p_{j} \mathrm{~d} m_{1}-\int p_{j} \mathrm{~d} m_{2}\right| .
$$

Setting $m_{1}=\Psi_{n}(u), m_{2}=\Psi_{n}(v)$, we deduce (2.18) from (2.14)-(2.16).

Corollary 2.2. Let the conditions of Theorem 2.3 be fulfilled. Then for all $l \in \mathbb{N}$ and $z_{1}, \ldots, z_{l} \in \mathbb{R}^{d}$

$$
\left(\widehat{\psi}_{n}\left(\cdot, z_{1}\right), \ldots, \widehat{\psi}_{n}\left(\cdot, z_{l}\right)\right) \stackrel{\mathrm{d}}{\longrightarrow}\left(\widehat{\psi}\left(\cdot, z_{1}\right), \ldots, \widehat{\psi}\left(\cdot, z_{l}\right)\right) .
$$

Corollary 2.3. Let a sequence $\left(\Psi_{n}\right)$ of probability-valued processes (PVP) satisfy conditions (2.10), (2.15), (2.16) and let for any $t \in \mathbb{R}_{+}$

$$
\lim _{z \rightarrow 0} U^{1}(t ; z)=1 .
$$

Then $U$ is the covaristic of some PVP $\Psi$ and $\Psi_{n} \stackrel{d}{\longrightarrow} \Psi$.

We denote $\Xi_{n}^{*}(t)=\sup _{u \leq t} \Xi_{n}(u)$ and introduce the conditions:

RC. For any $z$ the sequence $\left(\widehat{\psi}_{n}(\cdot, z)\right)$ is r.c. in C. 
UI. For any $t$ the sequence $\left(\Xi_{n}^{*}(t)\right)$ is uniformly integrable.

Taking to account that condition RC entails (2.16) and that relative compactness in $\mathrm{C}$ of a sequence of vector processes is a component-wise property ([7], VI.3.33), we can refine Corollary 2.2 as follows:

Corollary 2.4. Let conditions (2.10)-(2.15) and $\mathbf{R C}$ be fulfilled. Then the conclusion of Theorem 2.3 holds and for all $l \in \mathbb{N}, z_{1}, \ldots, z_{l} \in \mathbb{R}^{d}$

$$
\left(\widehat{\psi}_{n}\left(\cdot, z_{1}\right), \ldots, \widehat{\psi}_{n}\left(\cdot, z_{l}\right)\right) \stackrel{\mathrm{C}}{\longrightarrow}\left(\widehat{\psi}\left(\cdot, z_{1}\right), \ldots, \widehat{\psi}\left(\cdot, z_{l}\right)\right) .
$$

Below, l.i.p. signifies the limit in probability.

Theorem 2.4. Let a sequence $\left(\Psi_{n}\right)$ of MVP's satisfy conditions (2.10)-(2.15), RC and UI. Suppose that the characteristic function of the existing by Corollary $2.4 M V P \Psi$ such that $\Psi_{n} \stackrel{d}{\longrightarrow} \Psi$ and $\widetilde{\psi}=U$ satisfies, for any $t$, the relation

$$
\text { l.i.p. } \sup _{u \leq t} \operatorname{Re}(\widehat{\psi}(u, 0)-\widehat{\psi}(u, z))=0 \text {. }
$$

Then $\Psi_{n} \stackrel{\mathcal{C}}{\longrightarrow} \Psi$.

Proof. In view of Corollary 2.4 it suffices to show that the sequence $\left(\Psi_{n}\right)$ is r.c. in $\mathcal{C}$.

We fix $t$ and denote $S_{n}(u, z)=\operatorname{Re}\left(\widehat{\psi}_{n}(u, 0)-\widehat{\psi}_{n}(u, z)\right), S_{n}^{*}(z)=\sup _{u \leq t} S_{n}(u, z), K_{n}^{L}=\sup _{u \leq t} \psi_{n}\left(u, A_{L}\right)$, $B_{R}=\{x:|x|<R\}$ (in this proof, $|\cdot|$ is the sup-norm), $f_{R}(y)=\int_{B_{R}}\left(1-\mathrm{e}^{\mathrm{i} z y}\right) \mathrm{d} z$. The $j$ th component of $y \in \mathbb{R}^{d}$ will be denoted $y^{j}$.

Obviously,

$$
f_{R}(y)=(2 R)^{d}\left(1-\prod_{j=1}^{d} \frac{\sin R y^{j}}{R y^{j}}\right),
$$

so that $f_{R}$ assumes only real nonnegative values and

$$
f_{R}(y)>2^{d-1} R^{d} \quad \text { as } \quad|y|>2 / R .
$$

By Fubini's theorem and due to nonnegativeness of $f_{R}$

$$
\int_{B_{R}}\left(\widehat{\psi}_{n}(u, 0)-\widehat{\psi}_{n}(u, z)\right) \mathrm{d} z=\int f_{R}(y) \psi_{n}(u, \mathrm{~d} y) \geq \int_{A_{L}} f_{R}(y) \psi_{n}(u, \mathrm{~d} y),
$$

whence, setting $R=2 / L$, we get with account of $(2.21)$

$$
\int_{B_{2 / L}} S_{n}(u, z) \mathrm{d} z \geq 2^{2 d-1} L^{-d} \psi_{n}\left(u, A_{L}\right)
$$

Consequently,

$$
K_{n}^{L} \leq 2^{1-2 d} L^{d} \int_{B_{2 / L}} S_{n}^{*}(z) \mathrm{d} z
$$

The evident inequalities

$$
0 \leq S_{n}^{*}(z) \leq \Xi_{n}^{*}(t)
$$

together with condition UI allow to apply to (2.22) consecutively Fubini's and Fatou's theorems, which results in

$$
\varlimsup_{n \rightarrow \infty} \mathrm{E} K_{n}^{L} \leq 2^{1-2 d} L^{d} \int_{B_{2 / L}} \varlimsup_{n \rightarrow \infty} \mathrm{E} S_{n}^{*}(z) \mathrm{d} z .
$$


Obviously, the functional $\phi(f)=\sup _{u \leq t} \operatorname{Re} f(u)$ is continuous in the locally uniform topology and $S_{n}^{*}(z)=$ $\phi\left(\widehat{\psi}_{n}(\cdot, 0)-\widehat{\psi}_{n}(\cdot, z)\right), \Xi_{n}^{*}(t)=\phi\left(\widehat{\psi}_{n}(\cdot, 0)\right)$. Then it follows from Corollary 2.4 that

$$
\left(S_{n}^{*}(z), \Xi_{n}^{*}(t)\right) \stackrel{\mathrm{d}}{\longrightarrow}\left(S^{*}(z), \Xi^{*}(t)\right) \equiv(\phi(\widehat{\psi}(\cdot, 0)-\widehat{\psi}(\cdot, z)), \phi(\widehat{\psi}(\cdot, 0))),
$$

whence on the strength of (2.23) and UI

$$
S^{*}(z) \leq \Xi^{*}(t), \mathrm{E} \Xi^{*}(t)<\infty
$$

and $\varlimsup_{n \rightarrow \infty} \mathrm{E} S_{n}^{*}(z)=\mathrm{E} S^{*}(z)$. The last equality together with (2.24) yields

$$
\varlimsup_{n \rightarrow \infty} \mathrm{E} K_{n}^{L} \leq 2 \sup _{z \in B_{2 / L}} \mathrm{E} S^{*}(z)
$$

From (2.20) and (2.25) we deduce, by the dominated convergence theorem, that the right-hand side of the last inequality tends to zero as $L \rightarrow \infty$. So

$$
\lim _{L \rightarrow \infty} \varlimsup_{n \rightarrow \infty} \mathrm{P}\left\{\sup _{u \leq t} \psi_{n}\left(u, A_{L}\right)>\varepsilon\right\}=0
$$

for any $\varepsilon>0$. Now, relative compactness of $\left(\Psi_{n}\right)$ in $\mathcal{C}$ follows from $\mathbf{R C}$ by Corollary 1 in [24].

Corollary 2.5. Let a sequence $\left(\Psi_{n}\right)$ of PVP's satisfy conditions (2.10), (2.15), (2.19) and $\mathbf{R C}$ and let the characteristic function of the existing by Corollary 2.3 PVP $\Psi$ such that $\Psi_{n} \stackrel{d}{\longrightarrow} \Psi$ satisfy condition (2.20). Then $\Psi_{n} \stackrel{\mathcal{C}}{\longrightarrow} \Psi$.

Now, we are ready to apply the covaristics method. Another application - to empirical processes - was given in [23].

\section{A GEOMETRIC MODEL}

We will study from this time on sequences of MVP's of the level-exceedance type: $\Psi_{n}=\mu^{\xi_{n}}$, or, minutely,

$$
\psi_{n}(t, B)=\mu\left\{x: \xi_{n}(t, x) \in B\right\}, B \in \mathcal{B}^{\otimes d},
$$

where $\xi_{n}$ is an $\mathbb{R}^{d}$-valued varying random relief on $X$ and $\mu$ is a nonrandom finite measure on $\mathcal{X}$. Such processes are usual in stochastic geometry, to mention especially the coverage problem [5] studying geometric characteristics of $k$-multiple $\left(k \in \mathbb{Z}_{+}\right)$intersections of random sets. If this characteristic is measure, then we come to (3.1), where $\xi_{n}$ is a sum of indicators of arguments $t, x$ and $\omega$. If we ascribe, for physical or other reasons, to each covering set its thickness, then $\xi_{n}$ will be a finite linear combination of indicators. We may go farther and assume that $\xi_{n}$ is a sum of random "humps" and "hollows" which appear at random points of space and time, changing afterwards their location, size and, perhaps, shape. We will consider a model where shapes are fixed. It is described by the following assumptions.

$$
\text { A1. } X=\mathbb{R}^{p} \text {. }
$$

A2. $\xi_{n}(t, x)=\sum_{k: \tau_{n k} \leq t} F\left(\frac{n^{1 / p}\left(x-\zeta_{n k}-\eta_{n k}(t)\right)}{\rho_{n k}(t)}\right)$,

where $F: X \rightarrow \mathbb{R}^{d}$ is a nonrandom Borel function; $\left(\tau_{n k}, k \in \mathbb{N}\right)$ is a strictly increasing and a.s. tending to infinity sequence of positive random variables; $\zeta_{n k}, \eta_{n k}(t)$ and $\rho_{n k}(t)$ are random variables with values in $X, X$ and $\mathbb{R}_{+}$, respectively (if $\rho_{n k}(t)=0$, then, as the subsequent assumptions $\mathbf{A} \mathbf{5}$ and (3.3) will show, the respective summand equals zero for almost all $x$ ). All these random variables are given on a common probability space $\left(\Omega_{n}, \mathcal{F}_{n}, \mathrm{P}_{n}\right)$ (but in formulae we write $\mathrm{P}$ and $\mathrm{E}$ without subscript). 
A3. There exists a $\sigma$-algebra $\mathcal{F}_{n}^{0} \subset \mathcal{F}_{n}$ such that: (i) for all $k \in \mathbb{N}$ and $t>0$ the random variables $\tau_{n k}$ and $\eta_{n k}(t)$ are $\mathcal{F}_{n}^{0}$-measurable; (ii) for any $l \in \mathbb{N}$ and positive numbers $t_{1}, \ldots, t_{l}$ the random vectors $\left(\zeta_{n k}, \rho_{n k}\left(t_{1}\right), \ldots, \rho_{n k}\left(t_{l}\right)\right), k \in \mathbb{N}$, are conditionally w.r.t. $\mathcal{F}_{n}^{0}$ totally independent; (iii) for any $k \in \mathbb{N}$ and $t>0$ $\zeta_{n k}$ and $\rho_{n k}(t)$ are conditionally w.r.t. $\mathcal{F}_{n}^{0}$ independent.

A4. For each $k \zeta_{n k}$ has a conditional w.r.t. $\mathcal{F}_{n}^{0}$ distribution density $h_{n k}$.

A5. The measure $\mu$ has a bounded density w.r.t. the Lebesgue measure.

Remark 3.1. Similar models were studied in [19,21]. They feature with milder probabilistic assumptions but stricter geometric ones. In those models locations and sizes of "elementary reliefs" (the summands in A2) do not depend on time: $\eta_{n k}=0, \rho_{n k}(t)=\rho_{n k}$. The refusal from this assumption cardinally changes the emphasis and drastically complicates the rationale.

We introduce the notation: $\mathrm{E}^{0}=\mathrm{E}\left[\cdots \mid \mathcal{F}_{n}^{0}\right] \quad R_{n k}(t, A)=\mathrm{P}\left\{\rho_{n k}(t) \in A \mid \mathcal{F}_{n}^{0}\right\} \quad$ (the regular version),

$$
b_{n k}(t)=\mathrm{E}^{0} \rho_{n k}(t)^{p} \equiv \int_{0}^{\infty} r^{p} R_{n k}(t, \mathrm{~d} r), \quad N_{n}(t)=\#\left\{k: \tau_{n k} \leq t\right\}, \quad Q_{n}(t, x)=\frac{1}{n} \sum_{k=1}^{N_{n}(t)} b_{n k}(t) h_{n k}\left(x-\eta_{n k}(t)\right),
$$

$Q_{n}(t)=Q_{n}(t, \cdot), \xi_{n}(t)=\xi_{n}(t, \cdot), \mathbb{C}_{-}=\{\mathfrak{z} \in \mathbb{C}: \operatorname{Re} \mathfrak{z} \leq 0\}, \int=\int_{\mathbb{R}^{m}}(m$ will be determined by the context $)$, $\mathrm{M}^{l}=\int_{X^{l}} \cdots \mathrm{d} \mu^{\otimes l} \equiv \int \cdots \int \cdots \mu\left(\mathrm{d} x_{1}\right) \ldots \mu\left(\mathrm{d} x_{l}\right), \quad \mathbf{M}=\mathbf{M}^{1}$. Then (3.1) yields

$$
\widehat{\psi}_{n}(t, z)=\mathrm{Me}^{\mathrm{i} z \xi_{n}(t)} .
$$

This formula, otherwise written, was already used in the proof of Corollary 2.1.

Theorem 3.1. Let, for each $n \in \mathbb{N}$, an $M V P \Psi_{n}$ be defined by equality (3.1) and assumptions A1-A5. Suppose also that:

$$
\int|F(y)| \mathrm{d} y<\infty
$$

for each $k \in \mathbb{N}$ there exists an $\mathcal{F}_{n}^{0}$-measurable random variable $\vartheta_{n k}$ such that

$$
\sup _{x \in X} h_{n k}(x) \leq \vartheta_{n k}
$$

for each $k$ there exists a $\mathcal{B}_{+} \otimes \mathcal{F}_{n}^{0}$-measurable in $(r, \omega) \in \mathbb{R}_{+} \times \Omega_{n}$ random function $D_{n k}$ on $\mathbb{R}_{+}$such that for all $x^{\prime}, x^{\prime \prime} \in X$

$$
\left|h_{n k}\left(x^{\prime}\right)-h_{n k}\left(x^{\prime \prime}\right)\right| \leq D_{n k}\left(\left|x^{\prime}-x^{\prime \prime}\right|\right) ;
$$

for all $t>0, c>0, \varepsilon>0$ and $z \in \mathbb{R}^{d}$

$$
\begin{array}{r}
\sup _{s \leq t} \mathrm{P}\left\{\frac{1}{n} \sum_{k=1}^{N_{n}(s)} \vartheta_{n k} \int_{0}^{\infty} r^{p} R_{n k}(s, \mathrm{~d} r) \int_{|y|>c n^{1 / p} / r}|\sin z F(y)| \mathrm{d} y>\varepsilon\right\} \rightarrow 0, \\
\sup _{s \leq t}\left\{\frac{1}{n} \sum_{k=1}^{N_{n}(s)} \int_{0}^{\infty} r^{p} R_{n k}(s, \mathrm{~d} r) \int|\sin z F(y)| D_{n k}\left(\frac{r|y|}{n^{1 / p}}\right) \mathrm{d} y>\varepsilon\right\} \rightarrow 0, \\
\lim _{L \rightarrow \infty} \varlimsup_{n \rightarrow \infty} \sup _{s \leq t} \mathrm{P}\left\{\sum_{k=1}^{N_{n}(s)} \vartheta_{n k} b_{n k}(s)>n L\right\}=0 ;
\end{array}
$$


for all $l \in \mathbb{N}, t_{1}, \ldots, t_{l} \in \mathbb{R}_{+}$and $s_{1}, \ldots, s_{l} \in \mathbb{R}$

$$
\begin{aligned}
& \mathrm{E} \prod_{j=1}^{l} \mathrm{Me}^{\mathrm{i} s_{j} Q_{n}\left(t_{j}\right)} \rightarrow Z^{l}\left(t_{1}, \ldots, t_{l} ; \mathrm{i} s_{1}, \ldots, \mathrm{i} s_{l}\right), \\
& \lim _{\substack{c_{1}, \ldots, c_{l} \in \mathbb{R}, c_{1} \rightarrow 0, \ldots, c_{l} \rightarrow 0}} Z^{l}\left(t_{1}, \ldots, t_{l} ; \mathrm{i} c_{1}, \ldots, \mathrm{i} c_{l}\right)=\mu(X)^{l} ;
\end{aligned}
$$

for any $\mathfrak{z}_{1}, \mathfrak{z}_{2} \in \mathbb{C}_{-}$and $t>0$

$$
\lim _{n \rightarrow \infty} \sup _{0 \leq t_{1} \leq t_{2} \leq t}\left|\mathrm{E} \prod_{j=1}^{2} \mathrm{Me}^{\mathfrak{z}^{j} Q_{n}\left(t_{j}\right)}-Z^{2}\left(t_{1}, t_{2} ; \mathfrak{z}_{1}, \mathfrak{z}_{2}\right)\right|=0
$$

and the function $Z^{2}\left(\cdot, \cdot ; \mathfrak{z}_{1}, \mathfrak{z}_{2}\right)$ is continuous. Then:

(1) there exists an $\mathbb{R}_{+}$-valued random varying relief $Q$ on $X$ such that for all $l \in \mathbb{N}, t_{1}, \ldots, t_{l} \in \mathbb{R}_{+}$and $\mathfrak{z}_{1}, \ldots, \mathfrak{z}_{l} \in \mathbb{C}_{-}$

$$
Z^{l}\left(t_{1}, \ldots, t_{l} ; \mathfrak{z}_{1}, \ldots, \mathfrak{z}_{l}\right)=\mathrm{E} \prod_{j=1}^{l} \mathrm{Me}^{\mathfrak{z}^{j} Q\left(t_{j}\right)}
$$

(2) $\Psi_{n} \stackrel{d}{\longrightarrow} \Psi$, where

$$
\widehat{\psi}(t, z)=\mathrm{M} \exp \left\{Q(t) \int\left(\mathrm{e}^{\mathrm{i} z F(y)}-1\right) \mathrm{d} y\right\} .
$$

Theorem 3.2. Let, for each n, an $M V P \Psi_{n}$ be defined by equality (3.1) and assumptions A1-A5. Suppose that conditions (3.3)-(3.10) and $\mathbf{R C}$ are fulfilled. Then $\Psi_{n} \stackrel{\mathcal{C}}{\longrightarrow} \Psi$, where $\Psi$ and $Q$ are the same as in Theorem 3.1 .

The comparison of conditions (3.9) and (3.10) with similar conditions (2.10) and (2.13) of Theorem 2.3 and Corollaries 2.2-2.5 reveals the gist of Theorems 3.1 and 3.2: they reduce the problem we solve for the MVP's $\Psi_{n} \equiv \mu^{\xi_{n}}$ to a similar problem for the MVP's $\mu^{Q_{n}}$. The latter is much simpler, because so are the reliefs $Q_{n}$ in comparison with the $\xi_{n}$ 's and, besides, we do not impose on $\left(Q_{n}\right)$ the analogues of conditions $(2.15)$ and $(2.16)$. Herein the sequences $\left(\xi_{n}\right)$ and $\left(Q_{n}\right)$ are not asymptotically close in any customary sense. Indeed, the discontinuities of $\xi_{n}(\cdot, x)$ need not be small, whereas the processes $Q_{n}(\cdot, x)$ are, under rather general assumptions, asymptotically continuous. It will be seen from the proof of Theorem 3.1 that $Q_{n}(t, x)$ is an asymptotic equivalent (w.r.t. the proximity in probability) of $\mathrm{E}^{0} \xi_{n}(t, x)$.

The proofs of both theorems will be adduced in Section 4. And our present goal is to prove three ancillary statements facilitating the verification of their conditions.

Lemma 3.1. Let $\left\{h_{n}\right\}$ be a set of nonnegative Borel functions on $\mathbb{R}^{p}$ such that $\sup _{n} \int h_{n}(x) \mathrm{d} x<\infty$ and

$$
\sup _{n} \sup _{|x-y| \leq r}\left|h_{n}(x)-h_{n}(y)\right|<\infty
$$

for some $r>0$. Then there exists a constant $C$ such that for all $n$ and $x$

$$
h_{n}(x) \leq C
$$

Proof. $h_{n}(x)=\left(\int_{|y-x|<r}\left(h_{n}(x)-h_{n}(y)\right) \mathrm{d} y+\int_{|y-x|<r} h_{n}(y) \mathrm{d} y\right) / \int_{|y|<r} \mathrm{~d} y$.

Lemma 3.2. Let, for each $n, N_{n}$ be a counting process with compensator $\Lambda_{n}$, and let the sequence $\left(\Lambda_{n} / n\right)$ be r.c. in C. Then so is $\left(N_{n} / n\right)$. 
Proof. Denote $\breve{N}_{n}=N_{n}-\Lambda_{n}$. This is a local square integrable martingale such that for all $v \geq u \geq 0$

$$
\left\langle\breve{N}_{n}\right\rangle(v)-\left\langle\breve{N}_{n}\right\rangle(u) \leq \Lambda_{n}(v)-\Lambda_{n}(u)
$$

(equality is attained if $\Lambda_{n}$ is continuous). This inequality and relative compactness of $\left(\Lambda_{n} / n\right)$ in $\mathrm{C}$ imply, by Propositions VI.3.26 and VI.3.9 in [7], that the sequence $\left(\left\langle\breve{N}_{n} / n\right\rangle\right)$ is r.c. in C, too. Then by Rebolledo's theorem ([7], VI.4.13) the sequence $\left(\breve{N}_{n} / n\right)$ is r.c. in D. Hence, writing $N_{n}=\breve{N}_{n}+\Lambda_{n}$, we conclude by Lemma VI.3.31 [7] that the sequence $\left(N_{n} / n\right)$ is also r.c. in D. But all jumps of $N_{n} / n$ are of size $1 / n$, so Proposition VI.3.26 in [7] asserts that this sequence is r.c. in $\mathrm{C}$, as well.

To apply Theorem 3.2 one must be able to check condition RC. Since $\left|\widehat{\psi}_{n}(t, z)\right|=1$, it is equivalent, by Proposition VI.3.26 [7], to the relation

$$
\lim _{c \rightarrow 0} \varlimsup_{n \rightarrow \infty} \mathrm{P}\left\{\sup _{(v-c)_{+}<u<v \leq t}\left|\widehat{\psi}_{n}(v, z)-\widehat{\psi}_{n}(u, z)\right|>\varepsilon\right\}=0,
$$

where $t$ and $\varepsilon$ are arbitrary positive numbers and $z$ is an arbitrary row vector in $\mathbb{R}^{d}$. The next statement reduces it to simpler ones.

Henceforth, $a$ stands for $1 / p$. Also, we denote $F_{n k}(t, x)=F\left(\frac{n^{a}\left(x-\zeta_{n k}-\eta_{n k}(t)\right)}{\rho_{n k}(t)}\right)$, $F_{n k}(t)=F_{n k}(t, \cdot), \Phi_{n}(u, t)=\sum_{\tau_{n k} \leq u} F_{n k}(t)$ (so that $\left.\xi_{n}(t)=\Phi_{n}(t, t)\right)$.

Lemma 3.3. Let, for each $n \in \mathbb{N}$, an $M V P \Psi_{n}$ be defined by equality (3.1) and assumptions $\mathbf{A 1}, \mathbf{A 2}$ and $\mathbf{A 5}$. Suppose also that for some $z \in \mathbb{R}^{d}, t>0$ and any $\varepsilon>0$

$$
\begin{aligned}
& \int\left|\sin \frac{z F(y)}{2}\right| \mathrm{d} y<\infty, \\
& \lim _{c \rightarrow 0} \varlimsup_{n \rightarrow \infty} \mathrm{P}\left\{\sup _{(v-c)_{+}<u<v \leq t} \sum_{u<\tau_{n k} \leq v} \rho_{n k}(v)^{p}>n \varepsilon\right\}=0, \\
& \lim _{c \rightarrow 0} \varlimsup_{n \rightarrow \infty} \mathrm{P}\left\{\sup _{(v-c)_{+}<u<v \leq t}\left|\mathrm{Me}^{\mathrm{i} z \Phi_{n}(u, v)}-\mathrm{Me}^{\mathrm{i} z \Phi_{n}(u, u)}\right|>\varepsilon\right\}=0 .
\end{aligned}
$$

Then relation (3.16) holds for these $z, t$ and all $\varepsilon$.

Proof. The identity

$$
\mathrm{e}^{\mathrm{i} z \xi_{n}(v)}-\mathrm{e}^{\mathrm{i} z \xi_{n}(u)}=\mathrm{e}^{\mathrm{i} z \Phi_{n}(u, v)}\left(\exp \left\{\mathrm{i} z \sum_{u<\tau_{n k} \leq v} F_{n k}(v)\right\}-1\right)+\mathrm{e}^{\mathrm{i} z \Phi_{n}(u, v)}-\mathrm{e}^{\mathrm{i} z \Phi_{n}(u, u)}
$$

together with (3.2) yields

$$
\left|\widehat{\psi}_{n}(v, z)-\widehat{\psi}_{n}(u, z)\right| \leq \sum_{u<\tau_{n k} \leq v} \mathrm{M}\left|\mathrm{e}^{\mathrm{i} z F_{n k}(v)}-1\right|+\left|\mathrm{Me}^{\mathrm{i} z \Phi_{n}(u, v)}-\mathrm{Me}^{\mathrm{i} z \Phi_{n}(u, u)}\right| .
$$


Let $f$ denote the existing by assumption A5 Lebesgue density of $\mu$. By the same assumption $f$ is bounded. Denote also $\sigma_{n k}(t)=\zeta_{n k}+\eta_{n k}(t)$. Then

$$
\begin{aligned}
\mathrm{M}\left|\mathrm{e}^{\mathrm{i} z F_{n k}(v)}-1\right| & =2 \mathrm{M} \int\left|\sin \left(\frac{z}{2} F\left(\frac{n^{a}\left(x-\sigma_{n k}(v)\right)}{\rho_{n k}(v)}\right)\right)\right| f(x) \mathrm{d} x \\
& =\frac{2 \rho_{n k}(v)^{p}}{n} \int\left|\sin \frac{z F(y)}{2}\right| f\left(\sigma_{n k}(v)+\frac{\rho_{n k}(v) y}{n^{a}}\right) \mathrm{d} y
\end{aligned}
$$

which jointly with (3.20)-(3.17) and boundedness of $f$ implies (3.16).

The first of three examples illustrates Theorem 3.1. Assumptions A1-A5 enter (maybe, in a sharpened form stated explicitly) all of them. We consider also, for explanatory purposes, that $\mu(X)=1$.

Example 3.1. Let $R_{n k}(t, A)=R(A), h_{n k}(x)=h_{n}(x)$, where $R$ is a nonrandom measure and $h_{n}$ is a deterministic function. Assume so far (further we shall demand more) that

$$
\lim _{L \rightarrow \infty} \varlimsup_{n \rightarrow \infty} \mathrm{P}\left\{N_{n}(t)>n L\right\}=0
$$

and the functions $h_{n}$ are equicontinuous:

$$
\lim _{r \rightarrow 0} \sup _{n} \sup _{|x-y| \leq r}\left|h_{n}(x)-h_{n}(y)\right|=0 .
$$

Then by Lemma 3.1 they are uniformly bounded. So, to verify condition (3.6) it suffices to show that for any $c>0$

$$
\int_{0}^{\infty} r^{p} R(\mathrm{~d} r) \int_{|y|>c n^{a} / r}|F(y)| \mathrm{d} y \rightarrow 0 .
$$

And this will follow from (3.3) and Lebesgue's dominated convergence theorem applied twice if we demand that

$$
b \equiv \int_{0}^{\infty} r^{p} R(\mathrm{~d} r)<\infty .
$$

Relations (3.14) (a consequence of (3.22)), (3.21) and (3.23) imply (3.8) as well.

Denote $D_{n}(r)=\sup _{|x-y| \leq r}\left|h_{n}(x)-h_{n}(y)\right|$ (so that relation (3.5) holds automatically), $\delta_{n}(r)=$ $\int|F(y)| D_{n}\left(n^{-a} r|y|\right) \mathrm{d} y$. Due to (3.22) (and hereon (3.14)) $D_{n}\left(n^{-a} r\right) \rightarrow 0$ for any $r$ and the sequence $\left(D_{n}\right)$ is uniformly bounded, which together with (3.3), (3.23) and Lebesgue's theorem applied twice yields

$$
\int_{0}^{\infty} r^{p} \delta_{n}(r) R(\mathrm{~d} r) \rightarrow 0
$$

Hence in view of (3.21) relation (3.7) follows.

To check (3.9)-(3.11) we assume that

$$
\eta_{n k}(t)=g\left(\tau_{n k},\left(t-\tau_{n k}\right)_{+}\right),
$$

where $g$ is a nonrandom Borel function. Then

$$
Q_{n}(t)=\frac{b}{n} \int_{0}^{t} q_{n}(\tau, t-\tau) \mathrm{d} N_{n}(\tau)
$$

where $q_{n}(\tau, u)=q_{n}(\tau, u, \cdot), q_{n}(\tau, u, x)=h_{n}\left(x-g\left(\tau, u_{+}\right)\right)$. 
We take an arbitrary bounded sequence $\left(u_{n}\right) \in \mathbb{R}_{+}^{\mathbb{N}}$ and introduce (partly replicate) the notation: $\Lambda_{n}$ - the compensator of $N_{n}, \breve{N}_{n}=N_{n}-\Lambda_{n}$,

$$
\widetilde{Q}_{n}(t)=\frac{b}{n} \int_{0}^{t} q_{n}(\tau, t-\tau) \mathrm{d} \Lambda_{n}(\tau), \alpha_{n}(t)=\frac{1}{n} \int_{0}^{t} q_{n}\left(\tau, u_{n}-\tau\right) \mathrm{d} \breve{N}_{n}(\tau) .
$$

The last process is a local square integrable martingale with quadratic characteristic

$$
\left\langle\alpha_{n}\right\rangle(t)=\frac{1}{n^{2}} \int_{0}^{t} q_{n}\left(\tau, u_{n}-\tau\right)^{2} \mathrm{~d}\left\langle\breve{N}_{n}\right\rangle(\tau) \leq n^{-2} C^{2} \Lambda_{n}(t)
$$

(the inequality relies on (3.14) and (3.15)). So, if we demand that for any $t$

$$
\Lambda_{n}(t) / n^{2} \stackrel{\mathrm{P}}{\longrightarrow} 0,
$$

then Lenglart's inequality ([7], I.3.30)

$$
\mathrm{P}\left\{\sup _{v \leq t}\left|\alpha_{n}(v)\right| \geq \varepsilon\right\} \leq c \varepsilon^{-2}+\mathrm{P}\left\{\left\langle\alpha_{n}\right\rangle(t) \geq c\right\}
$$

and (3.25) will imply that, for any bounded sequence $\left(v_{n}\right), \alpha_{n}\left(v_{n}\right) \stackrel{\mathrm{P}}{\longrightarrow} 0$. Setting $v_{n}=u_{n}$, we get $Q_{n}\left(u_{n}\right)-$ $\widetilde{Q}_{n}\left(u_{n}\right) \stackrel{\mathrm{P}}{\longrightarrow} 0$, whence by Lebesgue's theorem

$$
\operatorname{ME}\left|\mathrm{e}^{\mathfrak{z} Q_{n}\left(u_{n}\right)}-\mathrm{e}^{\mathfrak{z} \widetilde{Q}_{n}\left(u_{n}\right)}\right| \rightarrow 0
$$

for all $\mathfrak{z} \in \mathbb{C}_{-}$. This together with the evident inequality

$$
\left|\prod_{j=1}^{l} \mathrm{Me}^{\mathfrak{j}^{j} Q_{n}\left(t_{j}\right)}-\prod_{j=1}^{l} \mathrm{Me}^{\mathfrak{z}_{j} \widetilde{Q}_{n}\left(t_{j}\right)}\right| \leq \sum_{j=1}^{l} \mathrm{M}\left|\mathrm{e}^{\mathfrak{z}^{j} Q_{n}\left(t_{j}\right)}-\mathrm{e}^{\mathfrak{z}_{j} \widetilde{Q}_{n}\left(t_{j}\right)}\right|,
$$

where $\mathfrak{z}_{j} \in \mathbb{C}_{-}$, shows that

$$
\sup _{t_{1} \leq \ldots \leq t_{l} \leq t}\left|\mathrm{E} \prod_{j=1}^{l} \mathrm{Me}^{\mathfrak{j}_{j} Q_{n}\left(t_{j}\right)}-\mathrm{E} \prod_{j=1}^{l} \mathrm{Me}^{\mathfrak{z}^{j} \widetilde{Q}_{n}\left(t_{j}\right)}\right| \rightarrow 0 .
$$

So, demanding that for any $l \in \mathbb{N}, s_{1}, \ldots, s_{l} \in \mathbb{R}, t \in \mathbb{R}_{+}$and $\mathfrak{z}_{1}, \mathfrak{z}_{2} \in \mathbb{C}_{-}$

$$
\mathrm{E} \prod_{j=1}^{l} \mathrm{Me}^{\mathrm{i} s_{j} \widetilde{Q}_{n}\left(t_{j}\right)} \rightarrow \mathrm{E} \prod_{j=1}^{l} \mathrm{Me}^{\mathrm{i} s_{j} Q\left(t_{j}\right)}
$$

and

$$
\lim _{n \rightarrow \infty} \sup _{t_{1} \leq t_{2} \leq t}\left|\mathrm{E} \prod_{j=1}^{2} \mathrm{Me}^{\tilde{z}_{j} \widetilde{Q}_{n}\left(t_{j}\right)}-\mathrm{E} \prod_{j=1}^{2} \mathrm{Me}^{\mathfrak{z}_{j} Q\left(t_{j}\right)}\right|=0,
$$

where $Q$ is some varying random relief, we guarantee the fulfilment of conditions (3.9)-(3.11). This ends the theoretic part of the example which can be summarized as follows: if $R_{n k}(t, \cdot)=R(\cdot), h_{n k}=h_{n}, R$ and $h_{n}$ are nonrandom, conditions (3.3), (3.21)-(3.24) (with nonrandom $g$ ) and (3.26)-(3.28) are fulfilled and, for each 
$\mathfrak{z} \in \mathbb{C}_{-}$, the random process $\mathrm{Me}^{\mathfrak{z} Q(\cdot)}$ is stochastically continuous, then the second statement of Theorem 3.1 holds (whereas the first one is incorporated into the assumptions). It remains to illustrate the fulfilment of conditions (3.27) and (3.28).

Assume that $g$ is continuous and there exists a random process $K$ such that

$$
n^{-1} \Lambda_{n} \stackrel{\mathrm{C}}{\longrightarrow} K \text {. }
$$

Denote

$$
\widetilde{B}_{n}(t, x)=\int_{0}^{t} h_{n}(x-g(\tau, t-\tau)) \mathrm{d} \Lambda_{n}(\tau) / n, B_{n}(t, x)=\int_{0}^{t} h_{n}(x-g(\tau, t-\tau)) \mathrm{d} K(\tau),
$$

$B_{n}(t)=B_{n}(t, \cdot)$ (likewise with $\sim$ ). It easily follows from (3.14), (3.29) and Theorem III.8.3 [12] that for any $t$

$$
\sup _{u \leq t} \mathrm{M}\left|\widetilde{B}_{n}(u)-B_{n}(u)\right| \stackrel{\mathrm{P}}{\longrightarrow} 0 .
$$

Hence, noting that: (1) $\widetilde{Q}_{n}=b \widetilde{B}_{n} ;(2)$ for any $\mathfrak{z} \in \mathbb{C}_{-}$and $t>0$ the functions $\mathrm{Me}^{\mathfrak{z} B_{n}}, n \in \mathbb{N}$, of argument $u \in[0, t]$ are equicontinuous, we conclude that relations (3.27) and (3.28) will hold if for all $t \in \mathbb{R}_{+}$and $s \in \mathbb{R}$ the sequence $\left(\mathrm{Me}^{\mathrm{i} s B_{n}(t)}\right)$ converges in probability to some limit $\mathfrak{f}(s, t)$ and l.i.p. $\mathfrak{f}(c, t)=1$. Lévy's continuity theorem allows to re-formulate this assumption in the more visual form:

$$
\mathrm{Me}^{\mathrm{i} s B_{n}(t)} \stackrel{\mathrm{P}}{\longrightarrow} \mathrm{Me}^{\mathrm{i} s B(t)},
$$

where, for each $t, B(t) \equiv B(t, \cdot)$ is a random function on $X$. This entails, together with (3.22), (3.29) and continuity of $g$, relations (3.27) and (3.28) with $Q(t)=b B(t)$.

Conditions (3.21) and (3.26) can be waived, once we have imposed (3.29). And this condition can, in turn, be substituted by the demand that for any $t n^{-1} \Lambda_{n}(t) \stackrel{\mathrm{P}}{\longrightarrow} K(t)$, where $K$ is a continuous process. Indeed, in this case, as was shown in [10], $\sup _{u \leq t}\left|n^{-1} \Lambda_{n}(u)-K(u)\right| \stackrel{\mathrm{P}}{\longrightarrow} 0$ for all $t$, whence (3.29) follows.

Now, we will exemplify Theorem 3.2.

Example 3.2. Let assumptions A1 and A2 hold with

$$
p=1, \quad F=\nu I_{[0,1]}, \quad \rho_{n k}(t)=1,
$$

where $\nu$ is a fixed $d$-dimensional vector. Then $F_{n k}(t, x)=\nu I_{\left[\sigma_{n k}(t), \sigma_{n k}(t)+1 / n\right]}(x)$ and, consequently, for any $s \geq u \geq 0$

$$
\mathrm{Me}^{\mathrm{i} z \Phi_{n}(u, s)}=1+\sum_{m=1}^{N_{n}(u)}\left(\mathrm{e}^{\mathrm{i} z \nu}-1\right)^{m} \sum_{1 \leq k_{1}<\ldots<k_{m} \leq N_{n}(u)} \varphi_{\mathbf{k}_{m}}^{n}(s),
$$

where $\mathbf{k}_{m}=\left(k_{1}, \ldots, k_{m}\right)$,

$$
\varphi_{\mathbf{k}_{m}}^{n}(s)=\mu\left(\bigcap_{j=1}^{m}\left[\sigma_{n k_{j}}(s), \sigma_{n k_{j}}(s)+1 / n\right]\right) .
$$

Denote $\alpha_{\mathbf{k}_{m}}^{n}(s)=\max _{1 \leq j \leq m} \sigma_{n k_{j}}(s), \beta_{\mathbf{k}_{m}}^{n}(s)=\min _{1 \leq j \leq m} \sigma_{n k_{j}}(s)+1 / n$, $I_{\mathbf{k}_{m}}^{n}(s)=I\left\{\alpha_{\mathbf{k}_{m}}^{n}(s)<\beta_{\mathbf{k}_{m}}^{n}(s)\right\}, \chi_{k k^{\prime}}^{n}(s)=I\left\{\left|\sigma_{n k}(s)-\sigma_{n k^{\prime}}(s)\right|<1 / n\right\}$. Obviously,

$$
I_{\mathbf{k}_{m}}^{n}(s) \leq \prod_{1 \leq i<j \leq m} \chi_{k_{i} k_{j}}^{n}(s)
$$


and the intersection of $\left[\sigma_{n k_{j}}(s), \sigma_{n k_{j}}(s)+1 / n\right], j=1, \ldots, m$, equals either $\left[\alpha_{\mathbf{k}_{m}}^{n}(s), \beta_{\mathbf{k}_{m}}^{n}(s)\right]$ (if this interval is nonvoid) or $\emptyset$ (otherwise). So, assuming $\mathbf{A 5}$, we can rewrite (3.33) in the form

$$
\varphi_{\mathbf{k}_{m}}^{n}(s)=I_{\mathbf{k}_{m}}^{n}(s) \int_{\alpha_{\mathbf{k}_{m}}^{n}(s)}^{\beta_{\mathbf{k}_{m}}^{n}(s)} f(x) \mathrm{d} x,
$$

whence

$$
\dot{\varphi}_{\mathbf{k}_{m}}^{n}(s)=I_{\mathbf{k}_{m}}^{n}(s)\left(f\left(\beta_{\mathbf{k}_{m}}^{n}(s)\right) \dot{\beta}_{\mathbf{k}_{m}}^{n}(s)-f\left(\alpha_{\mathbf{k}_{m}}^{n}(s)\right) \dot{\alpha}_{\mathbf{k}_{m}}^{n}(s)\right)
$$

provided the derivatives on the right-hand side exist.

Suppose further that

$$
\eta_{n k}(t)=t-\tau_{n k}
$$

and $f$ satisfies the Lipschitz condition

$$
|f(x)-f(y)| \leq C_{0}|x-y|
$$

with some constant $C_{0}$. Then $\sigma_{n k_{j}}(s)-\sigma_{n k_{1}}(s)=\zeta_{n k_{j}}-\zeta_{n k_{1}}-\tau_{n k_{j}}+\tau_{n k_{1}}, \alpha_{\mathbf{k}_{m}}^{n}(s)=s+\max _{1 \leq j \leq m}\left(\zeta_{n k_{j}}-\tau_{n k_{j}}\right)$, $\beta_{\mathbf{k}_{m}}^{n}(s)=s+\min _{1 \leq j \leq m}\left(\zeta_{n k_{j}}-\tau_{n k_{j}}\right)+1 / n$ as $s>\tau_{n k_{m}}$. These equalities together with (3.35), (3.34) and (3.37) yield for $v \geq u$

$$
\left|\varphi_{\mathbf{k}_{m}}^{n}(v)-\varphi_{\mathbf{k}_{m}}^{n}(u)\right| \leq \frac{C_{0}(v-u)}{n} \prod_{j=2}^{m} I\left\{\left|\zeta_{n k_{j}}-\zeta_{n k_{1}}-\tau_{n k_{j}}+\tau_{n k_{1}}\right|<\frac{1}{n}\right\}
$$

Assume the following:

A6. The random variables $\zeta_{n k}, k \in \mathbb{N}$, are independent of $\tau_{n k}, k \in \mathbb{N}$, and of each other.

A7. Each $\zeta_{n k}$ has a distribution density $h_{n}$, the same for all $k$.

Due to (3.36) and nonrandomness of $\rho_{n k}$ these assumptions entail $\mathbf{A 3}$ and $\mathbf{A 4}$ (one can take for $\mathcal{F}_{n}^{0}$ the $\sigma$-algebra generated by $\left.\tau_{n k}, k \in \mathbb{N}\right)$.

Let $\mathcal{G}_{n l}$ denote the $\sigma$-algebra generated by $\zeta_{n l}$ and $\tau_{n k}, k \in \mathbb{N}$. Then: assumption $\mathbf{A} \mathbf{6}$ implies that for any $k_{1}<\ldots<k_{m}$ and $\mathcal{G}_{n k_{1}}$-measurable random variables $\varpi_{2}, \ldots \varpi_{m}$

$$
\mathrm{E}\left(\prod_{j=2}^{m} I\left\{\left|\zeta_{n k_{j}}-\varpi_{j}\right|<\frac{1}{n}\right\} \mid \mathcal{G}_{n k_{1}}\right)=\prod_{j=2}^{m} \mathrm{P}\left\{\left|\zeta_{n k_{j}}-\varpi_{j}\right|<\frac{1}{n} \mid \mathcal{G}_{n k_{1}}\right\}
$$

assumptions $\mathbf{A 6}$ and $\mathbf{A 7}$ yield

$$
\mathrm{P}\left\{\left|\zeta_{n k_{j}}-\varpi_{j}\right|<1 / n \mid \mathcal{G}_{n k_{1}}\right\}=\int_{\varpi_{j}-1 / n}^{\varpi_{j}+1 / n} h_{n}(x) \mathrm{d} x .
$$

Supposing furthermore (3.14), we get from (3.38) and the last two equalities

$$
\mathrm{E}^{0} \sup _{(v-c)_{+}<u<v \leq t}\left|\varphi_{\mathbf{k}_{m}}^{n}(v)-\varphi_{\mathbf{k}_{m}}^{n}(u)\right| \leq C_{0} c(2 C)^{m-1} n^{-m} .
$$

And this together with (3.32) implies that

$$
\mathrm{E}^{0} \sup _{(v-c)_{+}<u<v \leq t}\left|\mathrm{Me}^{\mathrm{i} z \Phi_{n}(u, v)}-\mathrm{Me}^{\mathrm{i} z \Phi_{n}(u, u)}\right| \leq \frac{C_{0} c}{2 C} \sum_{m=1}^{N_{n}(t)}\left(\begin{array}{c}
N_{n}(t) \\
m
\end{array}\right)\left(\frac{4 C}{n}\right)^{m}=\frac{C_{0} c}{2 C}\left(\left(1+\frac{4 C}{n}\right)^{N_{n}(t)}-1\right) .
$$


So, postulating additionally (3.21), we provide (3.19). Condition (3.17) is in our case trivial, and condition (3.18) for $\rho_{n k}=1$ combined with (3.21) is tantamount to relative compactness of $\left(N_{n} / n\right)$ in C.

Now, taking to account that this example is a particular case of the previous one, we write down the complete

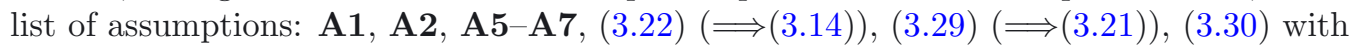

$$
B_{n}(t, x)=\int_{0}^{t} h_{n}(x-t+\tau) \mathrm{d} K(\tau)
$$

(3.31), (3.36), (3.37). If these assumptions are valid, then all the conditions of Lemmas 3.1-3.3 and Theorem 3.2 are fulfilled and therefore the conclusion of the theorem holds true. Since in our case $\mathrm{e}^{\mathrm{i} z F(y)}-1=$ $\left(\mathrm{e}^{\mathrm{i} z \nu}-1\right) I_{[0,1]}(y)$, equality $(3.13)$ acquires the more concrete form

$$
\widehat{\psi}(t, z)=\int \exp \left\{b B(t, x)\left(\mathrm{e}^{\mathrm{i} z \nu}-1\right)\right\} \mu(\mathrm{d} x) .
$$

So $\Psi(t)$ is a mixture (averaging in the spatial variable $x$ ) of Poisson distributions on the lattice $\nu \mathbb{Z}_{+}$.

Example 3.3. Let $\eta_{n k}(t)=0$ and

$$
\rho_{n k}(t)=\rho_{n k}^{0} T\left(t-\tau_{n k}\right),
$$

where the $\rho_{n k}^{0}$ 's are positive random variables and $T$ is a nonrandom positive increasing continuous function. Then denoting $\gamma_{n k}(x)=n^{a}\left(x-\zeta_{n k}\right) / \rho_{n k}^{0}$, we can write for $v \geq u \geq 0$ and $k \leq N_{n}(u)$

$$
\begin{aligned}
\mathrm{M}\left|\mathrm{e}^{2 \mathrm{i} z F_{n k}(v)}-\mathrm{e}^{2 \mathrm{i} z F_{n k}(u)}\right| & =2 \int\left|\sin z\left(F\left(\frac{\gamma_{n k}(x)}{T\left(v-\tau_{n k}\right)}\right)-F\left(\frac{\gamma_{n k}(x)}{T\left(u-\tau_{n k}\right)}\right)\right)\right| f(x) \mathrm{d} x \\
& =\frac{2}{n}\left(\rho_{n k}^{0}\right)^{p} \int\left|\sin z\left(F\left(\frac{y}{T\left(v-\tau_{n k}\right)}\right)-F\left(\frac{y}{T\left(u-\tau_{n k}\right)}\right)\right)\right| f\left(\zeta_{n k}+\frac{\rho_{n k}^{0} y}{n^{a}}\right) \mathrm{d} y
\end{aligned}
$$

whence, since $f$ is bounded,

$$
\mathrm{M}\left|\mathrm{e}^{2 \mathrm{i} z \Phi_{n}(u, v)}-\mathrm{e}^{2 \mathrm{i} z \Phi_{n}(u, u)}\right| \leq \frac{C}{n} \max _{k \leq N_{n}(u)} \int\left|\sin z\left(F\left(\frac{y}{T\left(v-\tau_{n k}\right)}\right)-F\left(\frac{y}{T\left(u-\tau_{n k}\right)}\right)\right)\right| \mathrm{d} y \sum_{k \leq N_{n}(u)}\left(\rho_{n k}^{0}\right)^{p}
$$

with some constant $C$. So, demanding that for any $t>0$ and $z \in \mathbb{R}^{d}$

$$
\lim _{L \rightarrow \infty} \varlimsup_{n \rightarrow \infty} \mathrm{P}\left\{\sum_{k=1}^{N_{n}(t)}\left(\rho_{n k}^{0}\right)^{p}>n L\right\}=0
$$

and

$$
\lim _{c \rightarrow 0} \sup _{(v-c)_{+}<u<v \leq t} \int\left|\sin z\left(F\left(\frac{y}{T(v)}\right)-F\left(\frac{y}{T(u)}\right)\right)\right| \mathrm{d} y=0
$$

we guarantee the fulfilment of condition (3.19) for all $t, \varepsilon$ and $z$. Condition (3.18) will be fulfilled, too, as equality (3.40) shows, if we demand additionally that the sequence $\left(N_{n} / n\right)$ be r.c. in C. And this is, by Lemma 3.2, a consequence of (3.29). So conditions (3.3), (3.29), (3.41) and (3.42) imply (3.17) - (3.19) and therefore, by Lemma 3.3, RC. 
Assume also $\mathbf{A} \mathbf{7}$ and a sharpened form of both $\mathbf{A} \mathbf{3}$ and $\mathbf{A 6}$ :

A8. The random variables $\zeta_{n k}, \rho_{n j}^{0}, k, j \in \mathbb{N}$, are independent of $\tau_{n k}, k \in \mathbb{N}$, and of each other. Suppose that the distribution of $\rho_{n k}^{0}$ does not depend on $n$ and $k$. Then

$$
R_{n k}(t, A)=R^{0}\left(A / T\left(t-\tau_{n k}\right)\right)
$$

$b_{n k}(t)=b^{0} T\left(t-\tau_{n k}\right)$ and

$$
Q_{n}(t, x)=b^{0} h_{n}(x) \int_{0}^{t} T(t-\tau) \mathrm{d} N_{n}(\tau) / n,
$$

where $R^{0}(A)=\mathrm{P}\left\{\rho_{n k}^{0} \in A\right\}, b^{0}=\mathrm{E}\left(\rho_{n k}^{0}\right)^{p}$. So, it is natural to demand instead of (3.23) that

$$
b^{0} \equiv \int_{0}^{\infty} r^{p} R^{0}(\mathrm{~d} r)<\infty .
$$

The last condition together with (3.14) and (3.21) implies, obviously, (3.8).

For all $t \geq s \geq 0$ and $k \leq N_{n}(s)$ we have, since $T$ increases, $T\left(s-\tau_{n k}\right) \leq T(t)$. Consequently, the left-hand side of (3.6) does not exceed

$$
\mathrm{P}\left\{T(t)^{p} N_{n}(t) \int_{0}^{\infty} r^{p} R^{0}(\mathrm{~d} r) \int|F(y)| I\left\{|y|>\frac{c n^{a}}{T(t) r}\right\} \mathrm{d} y>n \varepsilon\right\},
$$

which together with (3.3), (3.14), (3.21) and (3.45) yields, in the same way as in Example 3.1, relation (3.6). Likewise (3.7) follows from these conditions and the assumption that $T$ increases.

Finally, assume (3.29) and

A9. The functions $h_{n}$ regarded as random variables on the probability space $(X, \mathcal{X}, \mu)$ converge in distribution to some function $h$.

Then equality (3.44) where the function $T$ is, by assumption, continuous shows that relations (3.9), (3.10) and (3.12) with

$$
Q(t, x)=b^{0} h(x) \int_{0}^{t} T(t-\tau) \mathrm{d} K(\tau)
$$

hold for all values of the free variables.

As was noted above, condition (3.29) entails (3.21). This allows us to replace condition (3.41) with the following one:

$$
\forall m \in \mathbb{N} \quad \lim _{L \rightarrow \infty} \varlimsup_{n \rightarrow \infty} \mathrm{P}\left\{\sum_{k=1}^{m n}\left(\rho_{n k}^{0}\right)^{p}>n L\right\}=0 .
$$

The ultimate list of assumptions is: A1, A2, A5, A7-A9, $\eta_{n k}(t)=0,(3.3),(3.22),(3.29)$, (3.40) (with the subsequent explanations), (3.42), (3.43) and (3.47). If they are valid, then the conclusion of Theorem 3.2 holds true with $Q$ given by (3.46).

Remark 3.2. The model considered in Example 3.3 is a slight modification of the crystallization model studied by Kolmogorov [9]. In the latter, the rate of growth is at any instant the same for all crystals, whereas in the former it depends on the age of a crystal. Both cases are physically possible [25].

Remark 3.3. Rather general sufficient conditions for $\mathbf{R C}$ in a simpler but still meaningful model where $\eta_{n k}=0$ and $\rho_{n k}$ does not depend on time were obtained in $[19,21,24]$. 


\section{Proofs of Theorems 3.1 And 3.2}

Proof of Theorem 3.1. We will consider that $\mu(X)=1$ and $t_{1} \leq \ldots \leq t_{l}$, which, of course, does not diminish generality.

$1^{\circ}$. Denote $\Phi^{l}\left(t_{1}, \ldots, t_{l} ; s_{1}, \ldots, s_{l}\right)=Z^{l}\left(t_{1}, \ldots, t_{l} ; i s_{1}, \ldots, i s_{l}\right)$. Conditions (3.9) and (3.10) imply that the function $\Phi$ of variables $l \in \mathbb{N}, t_{1} \in \mathbb{R}_{+}, t_{2} \in \mathbb{R}_{+} \backslash\left\{t_{1}\right\}, \ldots, t_{l} \in \mathbb{R}_{+} \backslash\left\{t_{1}, \ldots, t_{l-1}\right\}, s_{1} \in \mathbb{R}, \ldots, s_{l} \in \mathbb{R}$ satisfies all the conditions of Theorem 2.2 which therefore asserts existence of a $\mathbb{P}(\mathbb{R})$-valued process $\Upsilon$ such that equality (2.6) holds for all values of the variables. By assumption A5 the measure $\mu$ is diffuse. Then Theorem 1.1 asserts existence of an $\mathbb{R}$-valued varying random relief $Q$ on $X$ such that, for all $B \in \mathcal{B}$,

$$
v(t, B)=\mu\{x: Q(t, x) \in B\}
$$

or, the same, $\widehat{v}(t, s)=\mathrm{Me}^{\mathrm{i} s Q(t)}$ for any $s \in \mathbb{R}$. Hence and from the definition of covaristic (formula (2.1)) we obtain

$$
\widetilde{v}^{l}\left(t_{1}, \ldots, t_{l} ; s_{1}, \ldots, s_{l}\right)=\mathrm{E} \prod_{j=1}^{l} \mathrm{Me}^{\mathrm{i} s_{j} Q\left(t_{j}\right)} .
$$

This together with (2.6) proves the first statement of the theorem.

$2^{\circ}$. Regarding $Q_{n}(\cdot)$ (here the dot marks the place for $t$ ) as a random process given on the probability space $\left(X^{\mathbb{N}} \otimes \Omega_{n}, \mathcal{X}^{\otimes \mathbb{N}} \otimes \mathcal{F}_{n}, \mu^{\otimes \mathbb{N}} \otimes \mathrm{P}_{n}\right)$, we see that relation (3.9) with right-hand side given by (3.12) asserts weak convergence of the finite-dimensional distributions of these processes to those of $Q(\cdot)$. Hence, taking to account nonnegativeness of all the $Q_{n}$ 's (and therefore $Q$ ), we deduce that for all $l \in \mathbb{N}, t_{1}, \ldots, t_{l} \in \mathbb{R}_{+}, \mathfrak{z}_{1}, \ldots, \mathfrak{z}_{l} \in \mathbb{C}_{-}$

$$
\mathrm{E} \prod_{j=1}^{l} \mathrm{Me}^{\mathfrak{z}_{j} Q_{n}\left(t_{j}\right)} \rightarrow \mathrm{E} \prod_{j=1}^{l} \mathrm{Me}^{\mathfrak{z}^{j} Q\left(t_{j}\right)}
$$

$3^{\circ}$. Denote $G(y, z)=\mathrm{e}^{\mathrm{i} z F(y)}-1, \mathfrak{g}(z)=\int G(y, z) \mathrm{d} y$ (the integral converges due to (3.3), and its value belongs to $\mathbb{C}_{-}$, because $\left.\cos z F(y)-1 \leq 0\right)$. Then equality $(3.12)$ shows that the covaristic of the MVP $\Psi$ with characteristic function (3.13) is given by the formula

$$
\widetilde{\psi}^{l}\left(t_{1}, \ldots, t_{l} ; z_{1}, \ldots, z_{l}\right)=Z^{l}\left(t_{1}, \ldots, t_{l} ; \mathfrak{g}\left(z_{1}\right), \ldots, \mathfrak{g}\left(z_{l}\right)\right) .
$$

Herein $\lim _{z \rightarrow 0} Z^{1}(t ; \mathfrak{g}(z))=1$ because of (3.12) and nonnegativeness of $Q$. So, to deduce the second statement from Corollary 2.3 and Theorem 2.1 it suffices to check the three conditions: (2.10) with

$$
U^{l}\left(t_{1}, \ldots, t_{l} ; z_{1}, \ldots, z_{l}\right)=Z^{l}\left(t_{1}, \ldots, t_{l} ; \mathfrak{g}\left(z_{1}\right), \ldots, \mathfrak{g}\left(z_{l}\right)\right)
$$

(2.15) and (2.16). In view of (4.1) and (3.12) condition (2.10) with this $U$ is tantamount to the relation

$$
\widetilde{\psi}_{n}^{l}\left(t_{1}, \ldots, t_{l} ; z_{1}, \ldots, z_{l}\right)-\mathrm{E} \prod_{j=1}^{l} \mathrm{Me}^{\mathfrak{g}\left(z_{j}\right) Q_{n}\left(t_{j}\right)} \rightarrow 0
$$

Having in mind verification of the other conditions, too, we will prove in items $4^{\circ}-11^{\circ}$ even more:

$$
\sup _{t_{1} \leq \ldots \leq t_{l} \leq t}\left|\widetilde{\psi}_{n}^{l}\left(t_{1}, \ldots, t_{l} ; z_{1}, \ldots, z_{l}\right)-\mathrm{E} \prod_{j=1}^{l} \mathrm{Me}^{\mathfrak{g}\left(z_{j}\right) Q_{n}\left(t_{j}\right)}\right| \rightarrow 0 .
$$


The notation $\chi_{n} \stackrel{\mathrm{M}^{l} \mathrm{P}}{\rightrightarrows} 0$, where $\chi_{1}, \chi_{2} \ldots$ are random functions on $[0, t]^{l} \times X^{l}$, will mean that, for any $\varepsilon>0$,

$$
\lim _{n \rightarrow \infty} \sup _{t_{1} \leq \ldots \leq t_{l} \leq t} M^{l} P\left\{\left|\chi_{n}\right|>\varepsilon\right\}=0
$$

In case the $\chi_{n}$ 's do not depend on $x_{1}, \ldots, x_{l}$ we write $\stackrel{\mathrm{P}}{\rightrightarrows}$.

$4^{\circ}$. Let us fix $l, z_{1}, \ldots, z_{l}$ and denote

$$
\begin{gathered}
S_{n}=\exp \left(\sum_{j=1}^{l} \mathfrak{g}\left(z_{j}\right) Q_{n}\left(t_{j}, x_{j}\right)\right), \xi_{n j}=\xi_{n}\left(t_{j}, x_{j}\right), \\
H_{n}=\mathrm{E}^{0} \prod_{j=1}^{l} \mathrm{e}^{\mathrm{i} z_{j} \xi_{n j}} .
\end{gathered}
$$

By construction

Because of (3.2)

$$
\left|S_{n}\right| \leq 1,\left|H_{n}\right| \leq 1
$$

$$
\prod_{j=1}^{l} \widehat{\psi}_{n}\left(t_{j}, z_{j}\right)=\mathrm{M}^{l} \prod_{j=1}^{l} \mathrm{e}^{\mathrm{i} z_{j} \xi_{n j}},
$$

which together with the definition of the covaristic and formula (4.3) yields

$$
\widetilde{\psi}_{n}^{l}\left(t_{1}, \ldots, t_{l} ; z_{1}, \ldots, z_{l}\right)=\mathrm{M}^{l} \mathrm{E} H_{n} .
$$

And this jointly with (4.4) shows that relation (4.2) is equivalent to

$$
\left|H_{n}-S_{n}\right| \stackrel{\mathrm{M}^{l} \mathrm{P}}{\rightrightarrows} 0
$$

$5^{\circ}$. We introduce the notation: $\rho_{n k j}=\rho_{n k}\left(t_{j}\right)$ (likewise $\left.\eta_{n k j}, b_{n k j}, R_{n k j}(\cdot)\right), F_{n k j}=F\left(\frac{n^{a}\left(x_{j}-\zeta_{n k}-\eta_{n k j}\right)}{\rho_{n k j}}\right) \equiv$ $F_{n k}\left(t_{j}, x_{j}\right)$ (recall that $\left.a=1 / p\right), \prod_{k}=\prod_{k=1}^{N_{n}\left(t_{l}\right)}$ (the same for sums), $J_{n k}=\left\{j \in\{1, \ldots, l\}: t_{j} \geq \tau_{n k}\right\} \equiv\{j \in$ $\left.\{1, \ldots, l\}: k \leq N_{n}\left(t_{j}\right)\right\}, \# J$ - the number of members in a finite set $J, \quad \sum_{J(2)}=\sum_{J \subset J_{n k}: \# J \geq 2}$ (the same for max),

$$
G_{n k j}=\mathrm{e}^{\mathrm{i} z_{j} F_{n k j}}-1 \equiv G\left(\frac{n^{a}\left(x_{j}-\zeta_{n k}-\eta_{n k j}\right)}{\rho_{n k j}}, z_{j}\right)
$$

$w_{n k}=\sum_{j \in J_{n k}} \mathrm{E}^{0} G_{n k j}, \Gamma_{n k}^{J}=\mathrm{E}^{0} \prod_{j \in J}\left|G_{n k j}\right|$. Obviously, for any summands

$$
\sum_{k} \sum_{j \in J_{n k}} \cdots=\sum_{j=1}^{l} \sum_{k=1}^{N_{n}\left(t_{j}\right)} \cdots
$$

By assumption A2 $\xi_{n j}=\sum_{k=1}^{N_{n}\left(t_{j}\right)} F_{n k j}$, whence

$$
\prod_{j=1}^{l} \mathrm{e}^{\mathrm{i} z_{j} \xi_{n j}}=\prod_{k} \prod_{j \in J_{n k}}\left(1+G_{n k j}\right) .
$$


This together with $\mathbf{A 3}$ (i, ii) yields another form of equality (4.3):

$$
H_{n}=\prod_{k}\left(1+w_{n k}+\sum_{J(2)} \mathrm{E}^{0} \prod_{j \in J} G_{n k j}\right) .
$$

Obviously, for an arbitrary natural number $m$ and any complex numbers $c_{1}, \ldots, c_{m}, d_{1}, \ldots, d_{m}$ such that $\left|c_{1}\right| \leq 1, \ldots,\left|c_{m}\right| \leq 1$ the inequality

$$
\left|\prod_{k=1}^{m} c_{k}-\prod_{k=1}^{m} d_{k}\right| \leq \sum_{k=1}^{m}\left(\left|c_{k}-d_{k}\right| \prod_{i=k+1}^{m}\left|d_{i}\right|\right)
$$

holds. Setting $m=N_{n}\left(t_{l}\right), c_{k}=\mathrm{E}^{0} \prod_{j \in J_{n k}} \mathrm{e}^{\mathrm{i} z_{j} F_{n k j}}$ (this is the $k$ th factor in (4.7)), $d_{k}=1+w_{n k}$, we get

$$
\left|H_{n}-\prod_{k}\left(1+w_{n k}\right)\right| \leq V_{n} W_{n},
$$

where $V_{n}=\sum_{k} \sum_{J(2)} \Gamma_{n k}^{J}$ and

$$
W_{n}=\prod_{k} \mathrm{e}^{\left|w_{n k}\right|}
$$

Now, our goal is to establish the relation

$$
\left|H_{n}-\prod_{k}\left(1+w_{n k}\right)\right| \stackrel{\mathrm{M}^{l} \mathrm{P}}{\rightrightarrows} 0 .
$$

It will be achieved if we show that

$$
V_{n} \stackrel{\mathrm{M}^{l} \mathrm{P}}{\rightrightarrows} 0
$$

and

$$
\lim _{L \rightarrow \infty} \varlimsup_{n \rightarrow \infty} \sup _{t_{1} \leq \ldots \leq t_{l} \leq t} M^{l} \mathrm{P}\left\{W_{n}>\mathrm{e}^{L}\right\}=0 .
$$

The proof of (4.11) is the subject of the next four items.

$6^{\circ}$. Let us fix $c>0$, denote

$$
\begin{gathered}
\gamma_{n k j}^{c}=\left|G_{n k j}\right| I\left\{\left|x_{j}-\eta_{n k j}-\zeta_{n k}\right|>c\right\}, \quad I_{n k}^{J c}=I\left\{\max _{i, j \in J}\left|x_{i}-\eta_{n k i}-\left(x_{j}-\eta_{n k j}\right)\right|>2 c\right\}, \\
V_{n}^{c}=\sum_{n} \sum_{J(2)} I_{n k}^{J c} \Gamma_{n k}^{J}
\end{gathered}
$$

and show that

$$
\sup _{t_{1} \leq \ldots \leq t_{l} \leq t} \mathrm{M}^{l} V_{n}^{c} \stackrel{\mathrm{P}}{\longrightarrow} 0 .
$$

Obviously, for any $y, y_{1}, \ldots, y_{l} \in X$ and nonvoid set $J \subset\{1, \ldots, l\}$ the inequality

$$
I\left\{\max _{i, j \in J}\left|y_{i}-y_{j}\right|>2 c\right\} \leq \sum_{j \in J} I\left\{\left|y_{j}-y\right|>c\right\}
$$


holds. Setting $y_{j}=x_{j}-\eta_{n k j}, y=\zeta_{n k}$ and taking to account that

$$
\left|G_{n k j}\right| \leq 2
$$

we get, for $J \subset J_{n k}$ such that $\# J \geq 2$,

$$
\mathrm{M}^{l} I_{n k}^{J c} \Gamma_{n k}^{J} \leq 2^{\# J-1} \sum_{j \in J} \mathrm{ME}^{0} \gamma_{n k j}^{c} .
$$

By assumptions $\mathbf{A 3}$ (i, iii) and $\mathbf{A} 4$ for any $k \in \mathbb{N}, s \geq \tau_{n k}, x \in X$ and bounded Borel function $\varphi: X \times \mathbb{R}_{+} \rightarrow \mathbb{C}$

$$
\mathrm{E}^{0} \varphi\left(x-\eta_{n k}(s)-\zeta_{n k}, \rho_{n k}(s)\right)=\int_{0}^{\infty} R_{n k}(s, \mathrm{~d} r) \int \varphi\left(x-\eta_{n k}(s)-y, r\right) h_{n k}(y) \mathrm{d} y .
$$

Hence, taking $\varphi(x, r)=g(\theta x / r) I\{|x|>c\}$, where $g: X \rightarrow \mathbb{C}$ is a bounded Borel function and $\theta$ is a positive parameter, and using the identity

$$
\int_{\left|y_{0}-y\right|>c} g\left(\frac{\theta\left(y_{0}-y\right)}{r}\right) f(y) \mathrm{d} y=\left(\frac{r}{\theta}\right)^{p} \int_{|y|>c \theta / r} g(y) f\left(y_{0}-\frac{r y}{\theta}\right) \mathrm{d} y,
$$

we get

$$
\begin{aligned}
& \mathrm{E}^{0} g\left(\frac{\theta\left(x-\eta_{n k}(s)-\zeta_{n k}\right)}{\rho_{n k}(s)}\right) I\left\{\left|x-\eta_{n k}(s)-\zeta_{n k}\right|>c\right\}= \\
& \theta^{-p} \int_{0}^{\infty} r^{p} R_{n k}(s, \mathrm{~d} r) \int_{|y|>c \theta / r} g(y) h_{n k}\left(x-\eta_{n k}(s)-\frac{r y}{n^{a}}\right) \mathrm{d} y .
\end{aligned}
$$

Setting here $\theta=n^{a}, g=\left|G\left(\cdot, z_{j}\right)\right|, x=x_{j}, s=t_{j}\left(j \in J_{n k}\right)$, we obtain with account of (3.4)

$$
\mathrm{E}^{0} \gamma_{n k j}^{c} \leq 2 \alpha_{n k j}^{c}
$$

where

$$
\alpha_{n k j}^{c}=\frac{\vartheta_{n k}}{n} \int_{0}^{\infty} r^{p} R_{n k j}(\mathrm{~d} r) \int_{|y|>c n^{a} / r}\left|\sin \frac{z_{j} F(y)}{2}\right| \mathrm{d} y .
$$

Noting that $\alpha_{n k j}^{c}$ does not depend on $x_{1}, \ldots, x_{l}$, we get from (4.13), (4.16) and (4.18)

$$
\mathrm{M}^{l} V_{n}^{c} \leq \sum_{k} \sum_{J \subset J_{n k}} 2^{\# J} \alpha_{n k j}^{c} .
$$

Obviously, for any finite set $M$ and numbers $u_{j}, j \in M$, the equality

$$
\sum_{J \subset M} \sum_{j \in J} u_{j}=2^{\# M-1} \sum_{j \in M} u_{j}
$$

holds. Consequently, for any nonnegative numbers $a_{J}$, where $J$ ranges over the subsets of $\{1, \ldots, l\}$,

$$
\sum_{k} \sum_{J \subset J_{n k}} a_{J} \sum_{j \in J} \alpha_{n k j}^{c} \leq 2^{l-1} \max _{J} a_{J} \sum_{k} \sum_{j \in J_{n k}} \alpha_{n k j}^{c} \stackrel{(4.6)}{=} 2^{l-1} \max _{J} a_{J} \sum_{j=1}^{l} \sum_{k=1}^{N_{n}\left(t_{j}\right)} \alpha_{n k j}^{c},
$$

which together with (4.20), (4.19) and (3.6) proves (4.14). 
$7^{\circ}$. Let us denote $\iota_{n k}^{c}=\max _{J(2)}\left(1-I_{n k}^{J c}\right), E_{n}=\left\{k: \# J_{n k} \geq 2\right\}, \bar{V}_{n}^{c}=\sum_{k} \sum_{J(2)}\left(1-I_{n k}^{J c}\right) \Gamma_{n k}^{J}$, so that

$$
V_{n}=V_{n}^{c}+\bar{V}_{n}^{c}
$$

and show that

$$
\mathrm{M}^{l} \bar{V}_{n}^{c} \leq 2^{2 l-1} \max _{k \in E_{n}} \mathrm{M}^{l} \iota_{n k}^{c} \cdot \sum_{j=1}^{l} \sum_{k=1}^{N_{n}\left(t_{j}\right)} \alpha_{n k j}^{0}
$$

Obviously,

$$
\bar{V}_{n}^{c} \leq \sum_{k} \iota_{n k}^{c} \sum_{\emptyset \neq J \subset J_{n k}} \Gamma_{n k}^{J}
$$

and $\left|G_{n k j}\right|=\gamma_{n k j}^{0}$. From the last equality, (4.15) and (4.18) we have $\Gamma_{n k}^{J} \leq 2^{\# J} \alpha_{n k j}^{0}$ for an arbitrary $j \in J$. Consequently,

$$
\Gamma_{n k}^{J} \leq 2^{l} \sum_{j \in J} \alpha_{n k j}^{0} \quad \text { for } J \neq \emptyset .
$$

The variables $x_{1}, \ldots, x_{l}$ do not enter, as is seen from (4.19), the right-hand side of this inequality. So, applying $\mathrm{M}^{l}$ to both sides of (4.24), we get

$$
\mathrm{M}^{l} \bar{V}_{n}^{c} \leq 2^{l} \max _{k \in E_{n}} \mathrm{M}^{l} \iota_{n k}^{c} \cdot \sum_{k} \sum_{\emptyset \neq J \subset J_{n k}} \sum_{j \in J} \alpha_{n k j}^{0} .
$$

Now, (4.23) follows from (4.21).

$8^{\circ}$. Let $f$ denote the existing by assumption $\mathbf{A} 5$ density of $\mu$. By the same assumption

$$
f(x) \leq C
$$

for some constant $C$. Let us show that for any $k \in E_{n}$

$$
\mathrm{M}^{l} \iota_{n k}^{c} \leq 2^{p-1} c^{p} l(l-1) C \int_{|y|<1} \mathrm{~d} y .
$$

Obviously,

$$
\iota_{n k}^{c}=I\left\{\min _{i, j \in J_{n k}, i \neq j}\left|x_{i}-\eta_{n k i}-\left(x_{j}-\eta_{n k j}\right)\right| \leq 2 c\right\} .
$$

So

$$
\mathrm{M}^{l} \iota_{n k}^{c} \leq \sum_{1 \leq i<j \leq l} \mu^{\otimes l}\left(A_{i j}^{(c)}\left(\eta_{n k i}, \eta_{n k j}\right)\right),
$$

where $A_{i j}^{(c)}\left(y^{\prime}, y^{\prime \prime}\right)=\left\{\left(x_{1}, \ldots, x_{l}\right):\left|x_{i}-y^{\prime}-\left(x_{j}-y^{\prime \prime}\right)\right| \leq 2 c\right\}$. The evident equalities

$$
\mu^{\otimes l}\left(A_{i j}^{(c)}\left(y^{\prime}, y^{\prime \prime}\right)\right)=\iint_{\left|x_{i}-x_{j}\right|<2 c} f\left(x_{i}+y^{\prime}\right) f\left(x_{j}+y^{\prime \prime}\right) \mathrm{d} x_{i} \mathrm{~d} x_{j}=2^{p} \iint_{|y|<c} f\left(x+y+y^{\prime}\right) f\left(x-y+y^{\prime \prime}\right) \mathrm{d} x \mathrm{~d} y
$$


and inequality (4.25) show that

$$
\mu^{\otimes l}\left(A_{i j}^{(c)}\left(y^{\prime}, y^{\prime \prime}\right)\right) \leq 2^{p} C \int_{|y|<c} \mathrm{~d} y \int f\left(x+y+y^{\prime}\right) \mathrm{d} x \equiv(2 c)^{p} C \int_{|y|<1} \mathrm{~d} y,
$$

which together with (4.27) proves (4.26).

$9^{\circ}$. From (4.19) we have

$$
\sum_{k=1}^{N_{n}\left(t_{j}\right)} \alpha_{n k j}^{0} \leq \frac{\left|z_{j}\right|}{n} \int|F(y)| \mathrm{d} y \sum_{k=1}^{N_{n}\left(t_{j}\right)} \vartheta_{n k} b_{n k}\left(t_{j}\right)
$$

whence because of $(4.23)$ and $(4.26)$

$$
\lim _{c \rightarrow 0} \varlimsup_{n \rightarrow \infty} \sup _{t_{1} \leq \ldots \leq t_{l} \leq t} \mathrm{P}\left\{\mathrm{M}^{l} \bar{V}_{n}^{c}>\varepsilon\right\}=0
$$

for any $\varepsilon>0$. This relation together with (4.14) and (4.22) implies that

$$
\mathrm{M}^{l} V_{n} \stackrel{\mathrm{P}}{\rightrightarrows} 0
$$

and, consequently, for any $r>0$

$$
\sup _{t_{1} \leq \ldots \leq t_{l} \leq t} \mathrm{E}\left(r \wedge \mathrm{M}^{l} V_{n}\right) \rightarrow 0
$$

Let $\varrho$ be an arbitrary nonnegative measurable random function on $X^{l} ; \varepsilon$ and $r$ be arbitrary positive numbers. Then the evident inequalities

show that

$$
\begin{gathered}
I\left\{\mathrm{M}^{l} \varrho>r\right\} \mathrm{M}^{l} I\{\varrho>\varepsilon\} \leq I\left\{\mathrm{M}^{l} \varrho>r\right\}, \\
I\left\{\mathrm{M}^{l} \varrho \leq r\right\} \mathrm{M}^{l} I\{\varrho>\varepsilon\} \leq \varepsilon^{-1} I\left\{\mathrm{M}^{l} \varrho \leq r\right\} \mathrm{M}^{l} \varrho
\end{gathered}
$$

$$
\mathrm{M}^{l} \mathrm{P}\{\varrho>\varepsilon\} \leq \mathrm{P}\left\{\mathrm{M}^{l} \varrho>r\right\}+\varepsilon^{-1} \mathrm{E}\left(r \wedge \mathrm{M}^{l} \varrho\right) .
$$

Putting $\varrho=V_{n}$, we deduce (4.11) from (4.28) and (4.29).

$10^{\circ}$. Let us prove (4.12), where $W_{n}$ is defined by (4.9). Recall that $w_{n k}=\sum_{j \in J_{n k}} \mathrm{E}^{0} G_{n k j}$.

Denote

$$
\varkappa_{n k}=\frac{1}{n} \sum_{j \in J_{n k}} \mathfrak{g}\left(z_{j}\right) b_{n k j} h_{n k}\left(x_{j}-\eta_{n k j}\right)
$$

$\left(\mathfrak{g}\right.$ was defined in item $\left.3^{\circ}\right)$. Setting in (4.17) $\theta=n^{a}, g=G\left(\cdot, z_{j}\right), x=x_{j}, s=t_{j}\left(j \in J_{n k}\right), c=0$, we get

$$
\mathrm{E}^{0} G_{n k j}=\frac{1}{n} \int_{0}^{\infty} r^{p} R_{n k j}(\mathrm{~d} r) \int G\left(y, z_{j}\right) h_{n k}\left(x_{j}-\eta_{n k j}-\frac{r y}{n^{a}}\right) \mathrm{d} y,
$$

which together with $(3.5)$ and the definition of $G$ (item $3^{\circ}$ ) yields

$$
\left|w_{n k}-\varkappa_{n k}\right| \leq \frac{1}{n} \sum_{j \in J_{n k}} \beta_{n k}\left(t_{j}, z_{j}\right)
$$

where

$$
\beta_{n k}(s, z)=2 \int_{0}^{\infty} r^{p} R_{n k}(s, \mathrm{~d} r) \int\left|\sin \frac{z F(y)}{2}\right| D_{n k}\left(\frac{r|y|}{n^{a}}\right) \mathrm{d} y .
$$

By the definition of $\varkappa_{n k}$ and condition (3.4)

$$
\left|\varkappa_{n k}\right| \leq \frac{C \vartheta_{n k}}{n} \sum_{j \in J_{n k}} b_{n k j},
$$


where $C=\max _{j \leq l}\left|\mathfrak{g}\left(z_{j}\right)\right|\left(<\infty\right.$ by condition (3.3)). This jointly with (4.6) and (4.31) implies that $\sum_{k}\left|w_{n k}\right| \leq$ $(C+1) T_{n} / n$, where

$$
T_{n}=\sum_{j=1}^{l} \sum_{k=1}^{N_{n}\left(t_{j}\right)}\left(\vartheta_{n k} b_{n k}\left(t_{j}\right)+\beta_{n k}\left(t_{j}, z_{j}\right)\right)
$$

This quantity does not depend on $x_{1}, \ldots, x_{l}$, so $\mathrm{M}^{l} \sum_{k}\left|w_{n k}\right| \leq(C+1) T_{n} / n$, too. Then from (4.9) and (4.30) we have for any $L, r>0$

$$
\sup _{t_{1} \leq \ldots \leq t_{l} \leq t} \mathrm{M}^{l} \mathrm{P}\left\{W_{n}>\mathrm{e}^{L}\right\}=\sup _{t_{1} \leq \ldots \leq t_{l} \leq t} \mathrm{M}^{l} \mathrm{P}\left\{\sum_{k}\left|w_{n k}\right|>L\right\} \leq \sup _{t_{1} \leq \ldots \leq t_{l} \leq t} \mathrm{P}\left\{T_{n}>\frac{r n}{C+1}\right\}+\frac{r}{L} .
$$

Equality (4.34) shows us that for any $\sigma>0$

$$
\sup _{t_{1} \leq \ldots \leq t_{l} \leq t} \mathrm{P}\left\{T_{n}>\sigma\right\} \leq \sum_{j=1}^{l}\left[\sup _{t_{j} \leq t} \mathrm{P}\left\{\sum_{k=1}^{N_{n}\left(t_{j}\right)} \vartheta_{n k} b_{n k}\left(t_{j}\right)>\frac{\sigma}{2 l}\right\}+\sup _{t_{j} \leq t} \mathrm{P}\left\{\sum_{k=1}^{N_{n}\left(t_{j}\right)} \beta_{n k}\left(t_{j}, z_{j}\right)>\frac{\sigma}{2 l}\right\}\right],
$$

which together with (4.35), (4.32) and (3.7) yields

$$
\lim _{L \rightarrow \infty} \varlimsup_{n \rightarrow \infty} \sup _{t_{1} \leq \ldots \leq t_{l} \leq t} \mathrm{M}^{l} \mathrm{P}\left\{W_{n}>\mathrm{e}^{L}\right\} \leq \sum_{j=1}^{l} \lim _{L \rightarrow \infty} \varlimsup_{n \rightarrow \infty} \sup _{t_{j} \leq t} \mathrm{P}\left\{\sum_{k=1}^{N_{n}\left(t_{j}\right)} \vartheta_{n k} b_{n k}\left(t_{j}\right)>\frac{n \mathrm{e}^{L}}{2 l(C+1)}\right\} .
$$

Now, (4.12) ensues from (3.8).

Once relations (4.11) and (4.12) together implying (4.10) have been proved, it remains (recall that we are proving (4.5)) to show that

$$
\prod_{k}\left(1+w_{n k}\right)-S_{n} \stackrel{\mathrm{M}^{l} \mathrm{P}}{\rightrightarrows} 0
$$

$11^{\circ}$. Writing, for arbitrary positive $\varepsilon$ and $L$,

$$
\mathrm{M}^{l} \mathrm{P}\left\{W_{n} \sum_{k}\left|w_{n k}-\varkappa_{n k}\right|>\varepsilon\right\} \leq \mathrm{M}^{l} \mathrm{P}\left\{W_{n}>\mathrm{e}^{L}\right\}+\mathrm{M}^{l} \mathrm{P}\left\{\sum_{k}\left|w_{n k}-\varkappa_{n k}\right|>\mathrm{e}^{-L} \varepsilon\right\},
$$

we deduce from (4.12), (4.31), (4.32) and (3.7) (the details are the same as in the previous item) that

$$
W_{n} \sum_{k}\left|w_{n k}-\varkappa_{n k}\right| \stackrel{\mathrm{M}^{l} \mathrm{P}}{\rightrightarrows} 0
$$

Obviously, $S_{n}=\prod_{k} \mathrm{e}^{\varkappa_{n k}}$. Noting that $\operatorname{Re} \mathfrak{g}\left(z_{j}\right) \leq 0, b_{n k j} \geq 0, h_{n k}(x) \geq 0$, we see that $\operatorname{Re} \varkappa_{n k} \leq 0$. Then, putting in (4.8) $m=N_{n}\left(t_{l}\right), c_{k}=\mathrm{e}^{\varkappa_{n k}}, d_{k}=1+w_{n k}$ and taking to account (4.9), we get

$$
\left|S_{n}-\prod_{k}\left(1+w_{n k}\right)\right| \leq W_{n} \sum_{k}\left|\mathrm{e}^{\varkappa_{n k}}-1-w_{n k}\right| .
$$

According to the maximum principle for analytic functions

$$
\sup _{\mathfrak{z} \in \mathbb{C}_{-}}\left|\frac{\mathrm{e}^{\mathfrak{z}}-1-\mathfrak{z}}{\mathfrak{z}^{2}}\right|=\sup _{s \in \mathbb{R}}\left|\frac{\mathrm{e}^{\mathrm{i} s}-1-i s}{s^{2}}\right|
$$


Hence, writing by Euler's and Taylor's formulae

$$
\mathrm{e}^{\mathrm{i} s}=1+i s-\left(\cos \theta_{1} s+i \sin \theta_{2} s\right) s^{2} / 2,
$$

we get $\left|\mathrm{e}^{\varkappa_{n k}}-1-\varkappa_{n k}\right| \leq\left|\varkappa_{n k}\right|^{2}$, which together with (4.33) and (4.6) yields

$$
\sum_{k}\left|\mathrm{e}^{\varkappa_{n k}}-1-\varkappa_{n k}\right| \leq \frac{C^{2}}{n^{2}}\left(\sum_{j=1}^{l} \sum_{k=1}^{N_{n}\left(t_{j}\right)} \vartheta_{n k} b_{n k}\left(t_{j}\right)\right)^{2} .
$$

Noting that the right-hand side of this inequality does not exceed

$$
\frac{C^{2} l}{n^{2}} \sum_{j=1}^{l}\left(\sum_{k=1}^{N_{n}\left(t_{j}\right)} \vartheta_{n k} b_{n k}\left(t_{j}\right)\right)^{2}
$$

we deduce (4.36) from (4.38), (4.37) and (3.8).

$12^{\circ}$. Let us check condition (2.15) (the set $A_{L}$ was defined before Theorem 2.3).

Denote $\kappa_{n}(s)=\left|\xi_{n}(s, \cdot)\right|, \kappa_{n}^{0}(s)=\mathrm{E}^{0} \kappa_{n}(s)$. Then

$$
\psi_{n}\left(s, A_{L}\right)=\mathrm{M} I\left\{\kappa_{n}(s)>L\right\}, \mathrm{E} \psi_{n}\left(s, A_{L}\right)=\operatorname{MP}\left\{\kappa_{n}(s)>L\right\},
$$

so (2.15) will follow from Chebyshev's inequality if we show that

$$
\lim _{r \rightarrow \infty} \varlimsup_{n \rightarrow \infty} \sup _{s \leq t} \operatorname{MP}\left\{\kappa_{n}(s)>r\right\}=0
$$

Writing $\mathrm{P}\{\kappa>r\} \leq \mathrm{E}\left(I\left\{\kappa_{n}^{0} \leq C\right\} \mathrm{E}^{0} I\left\{\kappa_{n}>r\right\}+I\left\{\kappa_{n}^{0}>C\right\}\right) \leq r^{-1} \mathrm{E} \kappa_{n}^{0} I\left\{\kappa_{n}^{0} \leq C\right\}+\mathrm{P}\left\{\kappa_{n}^{0}>C\right\} \leq$ $C / r+\mathrm{P}\left\{\kappa_{n}^{0}>C\right\}$ and then, on the basis of (4.30),

$$
\mathrm{MP}\left\{\kappa_{n}^{0}>C\right\} \leq \mathrm{P}\left\{\mathrm{M} \kappa_{n}^{0}>L\right\}+L / C,
$$

we reduce the task to the proof of the relation

$$
\lim _{L \rightarrow \infty} \varlimsup_{n \rightarrow \infty} \sup _{s \leq t} \mathrm{P}\left\{\mathrm{M} \kappa_{n}^{0}(s)>L\right\}=0 .
$$

By assumptions A1 and A3 (i)

$$
\kappa_{n}^{0}(s) \leq \sum_{k=1}^{N_{n}(s)} \epsilon_{n k}(s)
$$

where $\epsilon_{n k}(s)=\epsilon_{n k}(s, \cdot), \epsilon_{n k}(s, x)=\mathrm{E}^{0}\left|F\left(\frac{n^{a}\left(x-\zeta_{n k}-\eta_{n k}(s)\right)}{\rho_{n k}(s)}\right)\right|$. Taking in (4.17) $g=|F|, \theta=n^{a}, c=0$, we get

$$
\epsilon_{n k}(s, x)=\frac{1}{n} \int_{0}^{\infty} r^{p} R_{n k}(s, \mathrm{~d} r) \int|F(y)| h_{n k}\left(x-\eta_{n k}(s)-\frac{r y}{n^{a}}\right) \mathrm{d} y,
$$

which together with (4.40) and (3.4) yields

$$
\mathrm{M} \kappa_{n}^{0}(s) \leq \frac{1}{n} \sum_{k=1}^{N_{n}(s)} \vartheta_{n k} b_{n k}(s) \int|F(y)| \mathrm{d} y
$$


Now, (4.39) emerges from (3.3) and (3.8).

$13^{\circ}$. Let us check condition $(2.16)$.

Denote $\delta_{n}(u, v)=\widehat{\psi}_{n}(u, z)-\widehat{\psi}_{n}(v, z), \mathbf{t}=\left(t_{1}, t_{2}\right)$. Writing, for $\mathfrak{z}_{1}, \mathfrak{z}_{2} \in \mathbb{C}$,

$$
\left|\mathfrak{z}_{1}-\mathfrak{z}_{2}\right|^{2}=\mathfrak{z}_{1} \overline{\mathfrak{z}}_{1}-\mathfrak{z}_{1} \overline{\mathfrak{z}}_{2}-\mathfrak{z}_{2} \overline{\mathfrak{z}}_{1}+\mathfrak{z}_{2} \overline{\mathfrak{z}}_{2},
$$

setting $\mathfrak{z}_{1}=\widehat{\psi}_{n}(v, z)$ (so that $\left.\overline{\mathfrak{z}}_{1}=\widehat{\psi}_{n}(v,-z)\right), \mathfrak{z}_{2}=\widehat{\psi}_{n}(u, z)$ and recalling the definition of covaristic, we become convinced that

$$
\mathrm{E}\left|\delta_{n}(u, v)\right|^{2}=\widetilde{\psi}_{n}^{2}(v, v ; z,-z)-\widetilde{\psi}_{n}^{2}(v, u ; z,-z)-\left(\widetilde{\psi}_{n}^{2}(u, v ; z,-z)-\widetilde{\psi}_{n}^{2}(u, u ; z,-z)\right) .
$$

So it suffices to show that for any $t>0, z_{1}, z_{2} \in \mathbb{R}^{d}$

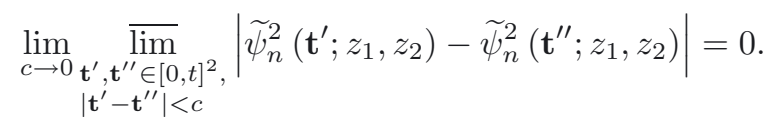

Writing on the basis of (4.2) and (3.11)

$$
\sup _{\mathbf{t} \in[0, t]^{2}}\left|\widetilde{\psi}_{n}^{2}\left(t_{1}, t_{2} ; z_{1}, z_{2}\right)-Z^{2}\left(t_{1}, t_{2} ; \mathfrak{g}\left(z_{1}\right), \mathfrak{g}\left(z_{2}\right)\right)\right| \rightarrow 0
$$

we deduce (4.41) from the assumed continuity of $Z^{2}\left(\cdot, \cdot ; \mathfrak{z}_{1}, \mathfrak{z}_{2}\right)$ and Cantor's theorem.

Proof of Theorem 3.2. The only distinction of the assumptions of this theorem from those of Theorem 3.1 is that condition (3.11) is substituted by RC. Noting that: firstly, (3.11) was used in the previous proof only for verification of condition (2.16); secondly, relation (2.19) with $U^{1}=\mathrm{E} \widehat{\psi}$ and relation (2.20) are immediate from (3.13) and (3.3), we deduce the desired conclusion from Corollary 2.5.

\section{REFERENCES}

[1] V. Beneš and J. Rataj, Stochastic Geometry: Selected Topics. Kluwer, Dordrecht (2004).

[2] D.J. Daley and D. Vere-Jones, An Introduction to the Theory of Point Processes. Elementary Theory and Methods. Springer, New York (2002) Vol. 1.

[3] D. Dawson, Measure-Valued Markov Processes. Lect. Notes Math. 1541 (1991).

[4] I.I. Gikhman and A.V. Skorokhod, Stochastic Differential Equations and Their Applications. Naukova Dumka, Kiev (1982) (Russian).

[5] P. Hall, Introduction to the Theory of Coverage Processes. Wiley, New York (1988).

[6] P.J. Huber, Robust Statistics. Wiley, New York (1981).

[7] J. Jacod and A.N. Shiryaev, Limit Theorems for Stochastic Processes. Springer, Berlin (1987).

[8] O. Kallenberg, Random Measures. Academic Press, New York, London; Akademie-Verlag, Berlin (1988).

[9] A.N. Kolmogorov, On the statistical theory of metal crystallization. Izvestiya Akademii Nauk SSSR [Bull. Acad. Sci. USSR] (1937), Issue 3, 355-359 (Russian) [English translation in: Selected Works of A.N. Kolmogorov, Probability Theory and Mathematical Statistics. Springer, New York (1992), Vol. 2, 188-192.

[10] D.L. McLeish, An extended martingale principle. Ann. Prob. 6 (1978) 144-150.

[11] Yu.V. Prokhorov, Convergence of random processes and limit theorems in probability theory. Th. Prob. Appl. 1 (1956) 157-214.

[12] A.N. Shiryaev, Probability. Springer, Berlin (1996)

[13] A.V. Skorokhod, Limit theorems for stochastic processes. Th. Prob. Appl. 1 (1956) 261-290.

[14] A.V. Skorokhod, Studies in the Theory of Random Processes. McGraw-Hill, New York (1965).

[15] A.V. Skorokhod, Stochastic Equations for Complex Systems. Kluwer, Dordrecht (1987).

[16] D. Stoyan, W.S. Kendall and J. Mecke, Stochastic Geometry and Its Applications. Akademie-Verlag, Berlin (1987).

[17] N.N. Vakhania, V.I. Tarieladze and S.A Chobanian, Probability Distributions in Banach Spaces. Reidel Pub. Co., DordrechtBoston (1987)

[18] A.P. Yurachkivsky, Covariance-characteristic functions of random measures and their applications to stochastic geometry. Dopovidi Natsionalnoŭi Akademii Nauk Ukrainy (1999), Issue 5, 49-54. 
[19] A.P. Yurachkivsky, Some applications of stochastic analysis to stochastic geometry. Th. Stoch. Proc. 5 (1999) $242-257$.

[20] A.P. Yurachkivsky, Covaristic functions of random measures and their applications. Th. Prob. Math. Stat. 60 (2000) $187-197$.

[21] A.P. Yurachkivsky, A generalization of a problem of stochastic geometry and related measure-valued processes. Ukr. Math. J. 52 (2000) 600-613.

[22] A.P. Yurachkivsky, Two deterministic functional characteristics of a random measure. Th. Prob. Math. Stat. 65 (2002) $189-197$.

[23] A.P. Yurachkivsky, Asymptotic study of measure-valued processes generated by randomly moving particles. Random Operators Stoch. Equations 10 (2002) 233-252.

[24] A. Yurachkivsky, A criterion for relative compactness of a sequence of measure-valued random processes. Acta Appl. Math. 79 (2003) 157-164.

[25] A.P. Yurachkivsky and G.G. Shapovalov, On the kinetics of amorphization under ion implantation, in: Frontiers in Nanoscale Science of Micron/Submicron Devices, NATO ASI, Series E: Applied Sciences, edited by A.-P. Jauho and E.V. Buzaneva. Kluwer, Dordrecht (1996) Vol. 328, 413-416.

[26] H. Zessin, The method of moments for random measures. Zeitschrift für Wahrscheinlichkeitstheorie und verwandte Gebiete 62 (1983) 359-409. 